NASA Technical Memorandum 100118

AIAA-87-1742

\title{
Method for the Determination of the Three- Dimensional Aerodynamic Field of a Rotor-Stator Combination in Compressible Flow
}

Sridhar M. Ramachandra

Case Western Reserve University

Cleveland, Ohio

Lawrence J. Bober

Lewis Research Center

Cleveland, Ohio

and

Suresh Khandelwal

Sverdrup Technology, Inc.

Lewis Research Center

Cleveland, Ohio

Prepared for the

23rd Joint Propulsion Conference

cosponsored by the AIAA, SAE, ASME, and ASEE

San Diego, California, June 29-July 2, 1987

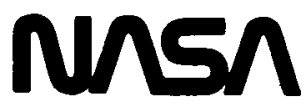

(AASA-TR-100118) BETECD FCE TEE

N $87-23625$

CEIEEHINATICN OF TEE TEREE-LILEASICAAL

AEECDYNAUIC FIEIL OF A EOTCE-STATCR

CCBBINATICN TC CCMERESSIEIE FICW (NASA)

F Avail: NTIS IC A03/EF A01 CSCL 21E G3/07 
METHOD FOR THE DETERMINATION OF THE THREE DIMENSIONAL AERODYNAMIC FIELD OF A ROTOR-STATOR COMBINATION IN COMPRESSIBLE FLOW

Sridhar M. Ramachandra

Case Western Reserve University

Cleveland, Ohio 44106

Lawrence J. Bober

National Aeronautics and Space Administration

Lewis Research Center

Cleveland, Ohio 44135

and

Suresh Khandelwal

Sverdrup Technology, Inc.

Lewis Research Center

Cleveland, Ohio 44135

\section{SUMMARY}

Using the lifting surface theory and the acceleration potential method for the flow field of an axial turbo-compressor stage, a recursive and a direct method are presented that make use of the eigenfunction solutions of the isolated rotor and stator to solve for the rotor-stator interaction problem. The net pressure distribution on the rotor and stator blades is represented by modified Birnbaum series, whose coefficients are determined using a matrix procedure and satisfying the boundary conditions on the surface of the blades. The relation between the matrix operators of the recursive and the direct methods is also shown. Expressions have been given for the blade circulation, the axial and tangential forces on the blade, the rotor power required, and the induced upwash velocity of the stage.

\section{INTRODUCTION}

The performance estimation of a turbomachine stage requires the aerodynamic characteristics of the rotor and the stator including their mutual interaction. The purpose of this paper is to discuss the aerodynamics of a rotor-stator combination in a turbomachine. The spacing between the adjacent blade rows of a turbomachine is generally small compared to the blade chord. Hence, the aerodynamic interference between the rotor and stator blade rows may be expected to be significant and needs to be included for an accurate analysis of the stage aerodynamic behavior. Existing theories like those of McCune (ref. 1), Namba (ref. 2), Schulten (ref. 3) and others consider only a single annular row of blades while other theories like that of Kajl and Okazaki (ref. 4) consider two adjacent two-dimensional infinite cascades. Hence, a method is required for calculating the aerodynamic flow field due to the the simultaneous presence of two rows of closely spaced annular cascades and is outlined in the following.

Since the chordwise and radial pressure distribution on the rotor and stator blades can be represented by a Birnbaum series, the heart of the lifting 
surface method is the determination of the coefficients of these series. In reference 5 , these coefficients were determined directly by satisfying flow tangency conditions on the upper and lower surfaces of the rotor and stator blades simultaneously. It was possible to obtain the parameters governing the blade loading and the discrete frequency noise field at the operating point.

In the following, a recursive scheme for determining the flow field by combining the solutions of the isolated rotor and stator will be outlined. Consequently, the procedure for calculating the off-design performance of a given rotor-stator combination is greatly simplified. The recursive procedure described here enables one to determine the Birnbaum coefficients of the rotorstator combination to any desired order of approximation. It is possible to estimate the aerodynamic interference between the two blade rows by this procedure.

A simple and direct method which solves for the flow field of the rotor and stator simultaneously is also indicated.

In section 2, a brief outline of the results of the acceleration potential method for dealing with the rotor-stator problem is given. In section 3 , the basic solutions to the rotor and stator are obtained by solving a nonhomogeneous problem giving the pressure distribution over the respective blades ignoring aerodynamic interference effects. These basic coefficients are modified to correct for the interference effects iteratively. The recurrence procedure used to calculate the Birnbaum series coefficients by satisfying the surface boundary conditions on the blades is discussed in section 3 which also contains a direct method for the same purpose. The estimation of the rotorstator aerodynamic interference is contained in section 4 . In section 5 , a discussion of the equations for obtaining the circulation distribution over the blades, the axial and tangential forces and their distribution, the shaft power absorbed by the rotor and the upwash field of the stage.

\section{EIGENFUNCTIONS OF THE ROTOR AND STATOR}

In this section, a brief procedure for obtaining the flow field of the rotor and stator using a distribution of acoustic monopole and dipole flow singularities on the blade surface is outlined. The rotor and stator with $\mathscr{Z}_{r}$ and $\mathscr{P}_{\mathrm{s}}$ blades, respectively, are considered to be situated in an unsteady, inviscid compressible flow field with the rotor placed upstream of the stator. The incoming flows for the rotor and stator are assumed to vary harmonically with the respective frequencies $\omega_{r}$ and $\omega_{s}$. The perturbations produced by the stage are considered to be small compared with the mean flow $W_{a}$ so that the linearized equations of motion in three dimensions may be used to represent the rotor and stator blades using suitable acoustic singularities. A coordinate system rotating with the rotor blades is chosen with respect to which the free stream has the mean velocity components

$$
\left(0, v_{r}, W_{r}\right)=\left(0, \Omega r, W_{a}\right)
$$

in the cylindrical coordinate system. The axial velocity $w_{a}$ is assumed to be constant through the stage. Relative to the rotating coordinate system 
fixed to the rotor, the inlet condition to the stator corresponds approximately to the value obtained from the stage velocity diagram and has the components

$$
\left(0, V_{s}, W_{s}\right)=\left(0, \Omega r-\tan \alpha_{2 r}, W_{a}\right)
$$

Since the chordwise and radial pressure distribution on the rotor and the stator blades is represented by a Birnbaum series, the heart of the lifting surface method is the determination of the coefficients of this series. In an earlier paper, the linearized aerodynamics of the combined rotor-stator system of an axial turbomachine for a uniform free stream using an acceleration potential representation was determined directly. A Green's function was introduced to satisfy the linearized differential equation for the rotor and stator. The radial variation of the Green's function for the rotor follows a Bessel's differential equation. For the stator, due to the presence of a constant swirl term $W_{a} \tan \alpha_{2 r}$ introduced by the rotor due to its exit blade angle $\alpha_{2 r}$. the radial variation of the Green's function is governed by a Whittaker differential equation. To make use of convenient orthogonality properties, this equation is transformed into the Laguerre differential equation. The Bessel and the generalized Laguerre and Kummer functions are used as the orthonormal basis functions to form the rotor and stator pressure eigenfunctions for the subsequent lifting surface theory. The rotor and stator blades are represented by a surface distribution of acoustic dipoles. The normalized radial pressure eigenfunction of the pulsating acoustic unit pressure monopoles on the rotor and stator may be shown (ref. 5) to be

$$
\begin{aligned}
& \Phi_{k \ell}\left(r_{1}\right)=\bar{A}(k, \ell) J_{k}\left(\lambda_{k \ell} r_{1}\right)+\bar{B}(k, \ell) Y_{k}\left(\lambda_{k \ell} r_{1}\right) \\
& \Psi_{\tilde{\ell} k \ell}\left(r_{1}\right)=\bar{C}(\tilde{l}, k, \ell) L_{\tilde{l}}^{(2 \bar{\alpha})}\left(r_{2}\right)+\bar{D} r_{2}^{-2 \bar{\alpha}}{ }_{1} F_{1}\left(-\tilde{l}-2 \bar{\alpha} ; 1-2 \bar{\alpha} ; r_{2}\right), r_{2}=v_{\tilde{\ell} k \ell} r_{1}
\end{aligned}
$$

where $\Phi_{k \ell}\left(r_{\eta}\right)$ is the normalized cylinder function in which $J_{k}(x), Y_{k}(x)$ are Bessel functions of the first and second kind, respectively; $\Psi_{\ell k \ell}(r \eta)$ is the normalized stator eigenfunction in whtch $L(b)(x)$ and $F_{1}(a, b ; x)$ are, respectively, the generalized Laguerre function and the Kummer function; $\underline{\lambda}_{k l}$ and $v_{\bar{l} k \ell}$ are the corresponding eigenvalues to be determined; $\bar{A}, \bar{B}, \bar{C}, \bar{D}$ are constants; $k=0, \pm 1, \pm 2, \ldots \ldots ; \ell=1,2,3, \ldots ; \ell=0,1,2,3, \ldots$. The eigenvalues $\lambda_{k \ell}$ and $v_{\tilde{l} k \ell}$ are obtained by satisfying the Neumann boundary conditions

$$
\begin{gathered}
d \Phi_{k \ell} / d r_{1}=0 \quad \text { at } \quad r_{1}=h_{r} \quad \text { and } 1 \\
d \Psi_{\ell k \ell} / d r_{1}=0 \quad \text { at } \quad r_{1}=R_{s r} h_{s} \quad \text { and } \quad R_{s r}
\end{gathered}
$$

at both the hub and tip of the rotor and stator. The pressure field of the surface distribution of pulsating unit pressure dipoles on the isolated rotor and stator blades is obtained by differentiating the monopole pressure function normal to the surface. The resulting pressure field for both the rotor and stator can be shown to be given by 


$$
\begin{aligned}
& P_{r}\left(\vec{r}_{1}, t_{1}\right)=-\frac{\mathscr{P}_{r} e^{1 \omega_{r} t}}{4 \pi \beta} \sum_{k_{r}} \sum_{\ell} \int_{h_{r}}^{1} \int_{z_{r}}^{z_{r} 2} \Delta P_{r} \Phi_{t}\left(\vec{r}_{1}, \vec{\rho}_{r}, t_{1} ; k_{r}, \ell\right) d \rho_{r} d \zeta_{r} \\
& P_{s}\left(\vec{r}_{1}, t_{1}\right)=\frac{\mathscr{Z}_{s} e^{i \omega_{s} t_{1}}}{8 \pi M M_{2}} \sum_{\tilde{l}} \sum_{k_{s}} \sum_{\ell} \int_{R_{s r} h_{s}}^{R_{s r}} \int_{s 1}^{z_{z} 2} \Delta P_{s} \Psi_{*}\left(\vec{r}_{1}, \vec{\rho}_{s}, t_{1} ; \tilde{l}, k_{s}, l\right) d \rho_{s} d \zeta_{s}
\end{aligned}
$$

where $\Delta P_{r}$ and $\Delta P_{S}$ are the net pressure distribution on the rotor and stator blades, respectively which are functions of $\left(\rho_{r}, \varphi_{r}, \zeta_{r}\right)$ and $\left(\rho_{S}, \varphi_{S}, \zeta_{S}\right)$. The functions $\Phi *$ and $\Psi *$ are defined by

$\Phi_{*}\left(\vec{r}_{1}, \vec{\rho}_{r}, t_{1} ; k_{r}, l\right)=\Phi_{k_{r} \ell}\left(\rho_{r}\right) \mathscr{F}_{20}\left(r_{1}\right) f_{2} \exp \left(i f_{0} \hat{\mathscr{X}}_{r}\right) \exp i\left[k_{r}\left(\theta-\bar{\varphi}_{r}-\varphi_{r}\right)\right]$

$\Psi_{*}\left(\vec{r}_{1}, \vec{\rho}_{s}, t_{1} ; \tilde{l}, k_{s}, l\right)=\mathscr{F}_{50}\left(\rho_{s}\right) \mathscr{F}_{50}\left(r_{1}\right) f_{5} \exp \left(-i \omega_{s}\left|\hat{\mathscr{X}}_{s}\right|\right) \exp 1\left[k_{s}\left(\theta-\bar{\varphi}_{s}-\varphi_{s}\right)\right]$

for subsonic axial flow, $M<1$. The pressure field $\mathrm{Pr}$ of the rotor has been obtained by summation over all the $\mathscr{X}_{r}$ rotor blades and 1 ikewise, the field of the stator has been obtained by summation over all the $\mathscr{Z}_{s}$ stator blades. The functions $f_{0}, f_{2}, \mathscr{F}_{20}$ and $f_{5}, \mathscr{F}_{50}$ are listed in the Appendix; $\hat{\mathscr{Z}}_{r}=z_{1}-\zeta_{r}$, $\mathscr{\mathscr { X }}_{s}=z_{1}-\zeta_{s}, \rho_{r}\left(\rho_{r}, \varphi_{r}, \zeta_{r}\right)$ and $\rho_{s}\left(\rho_{s}, \varphi_{s}, \zeta_{s}\right)$ are the respective locations of the dipoles on the rotor and stator blades.

The radial and chordwise distribution of pressure on a blade of the rotor and the stator is assumed to be given by the Glauert-Birnbaum series modulated radially by the corresponding radial eigenfunction. The Birnbaum series coefficients are assumed to be independent of the eigen-numbers $\tilde{l}, k, \ell$. Assuming that the thickness and camber effects are linearly superposable within the limits of the linear theory used here, the net pressure distribution on the rotor and stator blades can be written as

$$
\begin{aligned}
& \Delta P_{r}=H_{1}\left(\tilde{\omega}, \rho_{r} ; k_{r}, l\right)=\left(\mathscr{A}_{0} \cot \frac{\tilde{\omega}}{2}+\sum_{m=1}^{\infty} \mathscr{A}_{m} \sin \tilde{\omega}\right) \Phi_{k_{r} \ell}\left(\rho_{r}\right) \\
& \Delta P_{s}=H_{2}\left(\tilde{\omega}, \rho_{s} ; \tilde{l}, k_{s}, l\right)=\left(\mathscr{B}_{0} \cot \frac{\tilde{\omega}}{2}+\sum_{m=1}^{\infty} \mathscr{B}_{m} \sin \tilde{m}\right) \Psi_{\tilde{l} k l}\left(\rho_{s}\right)
\end{aligned}
$$


These are written in the matrix form

$$
\begin{gathered}
H_{1}\left(\tilde{\omega}, \rho_{r} ; k_{r}, l\right)=\pi \mathscr{A} \Phi_{k_{r} \ell}\left(\rho_{r}\right) \\
H_{2}\left(\tilde{\omega}, \rho_{s} ; \tilde{l}, k_{s}, l\right)=\pi \mathscr{B} \Psi_{\tilde{l}_{k_{s}} \ell}\left(\rho_{s}\right)
\end{gathered}
$$

where $\Pi, \mathscr{A}, \mathscr{B}$ denote the vectors

$$
\begin{gathered}
\Pi=\left(\cot \frac{\tilde{\omega}}{2}, \sin \tilde{\omega}, \sin 2 \tilde{\omega}, \ldots, \sin \tilde{\omega}, \ldots\right) \\
\mathscr{A}^{\top}=\left(\mathscr{O}_{0}, \mathscr{A}_{1}, \mathscr{A}_{2}, \ldots\right) \\
\mathscr{B}^{\top}=\left(\mathscr{D}_{0}, \mathscr{B}_{1}, \mathscr{B}_{2}, \ldots\right)
\end{gathered}
$$

The superscript $T$ indicates the transpose of the matrix and $\tilde{\omega}$ is the Glauert angle parameter for the chordwise position $y^{\prime}$, in the blade coordinate system shown in (fig. 1). The coordinates $y_{r}^{\prime}$ and $y_{s}^{\prime}$ of the rotor and stator are defined by

$$
\begin{array}{lll}
y_{r}^{\prime}=-C_{R} \cos \tilde{\omega}_{R} & -C_{r} \leq y_{r}^{\prime} \leq+C_{R} & 0 \leq \tilde{\omega}_{r} \leq \pi \\
y_{S}^{\prime}=+C_{S} \cos \tilde{\omega}_{S} & -C_{S} \leq y_{S}^{\prime} \leq+C_{S} & 0 \leq \tilde{\omega}_{S} \leq \pi
\end{array}
$$

In order to satisfy the boundary conditions on the blade surfaces, it is necessary to calculate the resultant velocity at a point on the blade surface. This is done by using the unsteady equation of motion in a helical coordinate system (fig. 2) and integrating the perturbation pressure along the undisturbed streamline direction (ref. 2) assuming that the pressure and the associated velocities also vary harmonically in time.

\section{THE BASIC SOLUTION}

The Birnbaum coefficient vectors introduced in section 2 are unknown and have to be determined by satisfying the appropriate conditions on the blade surfaces. The procedure for calculating the resultant velocity at any point in the flow field of the rotor and stator is described in this section. From this the blade surface conditions can be obtained. We shall denote $\mathscr{A}(0)$ and $\mathscr{B}(0)$ to be the basic Birnbaum coefficient vectors of the isolated rotor and stator, so that mutual interference effects are absent. In subsonic flow, these coefficients will be perturbed by aerodynamic interference effects when the rotor and stator are juxtaposed to form a stage. The resulting Birnbaum coefficients of rotor-stator combination will be denoted by $\mathscr{A}$ and $\mathscr{B}$. The basic coefficients $\mathscr{A}(0)$ and $\mathscr{D}(0)$ are determined by satisfying the surface boundary conditions on the rotor and stator blades. As the stator approaches the rotor, the induced velocity due to the stator disturbs the surface boundary conditions on the rotor. Hence, the rotor Birnbaum coefficients $\mathscr{N}(0)$ must be changed to 
satisfy the surface conditions again. Likewise, the stator Birnbaum coefficients $\mathscr{B}(0)$ must be changed due to the rotor interference field.

When the rotor and stator blades are kept in isolation, the resultant velocity $\vec{U}_{R}$ and $\vec{U}_{S}$ at the blade surface can written in the respective blade coordinate systems as

$$
\begin{aligned}
& \left(\begin{array}{l}
U_{R x^{\prime}} \\
U_{R y^{\prime}} \\
U_{R z^{\prime}}
\end{array}\right)=\left[\begin{array}{ccc}
\sin \theta & \cos \theta & 0 \\
\sin \alpha_{r} \cos \theta-\sin \alpha_{r} \sin \theta & \cos \alpha_{r} \\
-\cos \alpha_{r} \cos \theta & \cos \alpha_{r} \sin \theta & \sin \alpha_{r}
\end{array}\right]\left(\begin{array}{l}
\nu_{r r} \\
\varphi_{r \theta}+\cot \theta_{h 1} \\
\nu_{r z}+1
\end{array}\right) \\
& \left(\begin{array}{l}
U_{S x^{\prime}} \\
U_{S y^{\prime}} \\
U_{S z^{\prime}}
\end{array}\right)=\left[\begin{array}{ccc}
\sin \theta & \cos \theta & 0 \\
\sin \alpha_{r} \cos \theta-\sin \alpha_{r} \sin \theta & \cos \alpha_{s} \\
-\cos \alpha_{s} \cos \theta & \cos \alpha_{s} \sin \theta & \sin \alpha_{s}
\end{array}\right]\left(\begin{array}{l}
\varphi_{s r} \\
\psi_{s \theta}+\cot \theta_{h 2} \\
\psi_{s z}+A_{r s}^{\prime}
\end{array}\right) \\
& \mathscr{\nu}_{r r}=x_{r} \quad \Phi_{r \theta}=\left(x_{2} \cos \theta_{h 1}-x_{3} \sin \theta_{h 1}\right) d^{\top} \\
& \Phi_{r 2}=\left(x_{2} \sin \theta_{h 1}+x_{3} \cos \theta_{h 1}\right) \alpha^{\top} \\
& \mathscr{V}_{s r}=Y_{7} \mathscr{B}^{\top} \quad \mathscr{V}_{s \theta}=\left(Y_{2} \cos \theta_{h 2}-Y_{3} \sin \theta_{h 2}\right) \mathscr{B}^{\top} \\
& \nu_{s z}=\left(Y_{2} \sin \theta_{h 2}+Y_{3} \cos \theta_{h 2}\right) S
\end{aligned}
$$

where $\mathscr{A}$ and $\mathscr{B}$ are the vectors of Birnbaum coefficients and the functions $X_{1}, X_{2}, X_{3}, Y_{1}, Y_{2}, Y_{3}$ are listed below. 


$$
\begin{aligned}
& x_{1}=K_{r} \sum_{k} \sum_{\ell} \int_{z_{r 1}}^{z} \int_{h_{r}}^{1} \pi \Phi_{k \ell}^{2}\left(\rho_{r}\right) \mathscr{F}_{1} d \rho_{r} d \zeta_{r} \\
& x_{2}=k_{r} \sum_{k} \sum_{l} \int_{z_{r}}^{2} \int_{h_{r}}^{1} \pi \Phi_{k \ell}^{2}\left(\rho_{r}\right) \mathscr{F}_{2} d \rho_{r} d \zeta_{r} \\
& x_{3}=k_{r} \sum_{k} \sum_{\ell} \int_{z_{r 1}}^{z} \int_{h_{r}}^{1} \pi \Phi_{k \ell}^{2}\left(\rho_{r}\right) \mathscr{F}_{3} d \rho_{r} d \zeta_{r}
\end{aligned}
$$

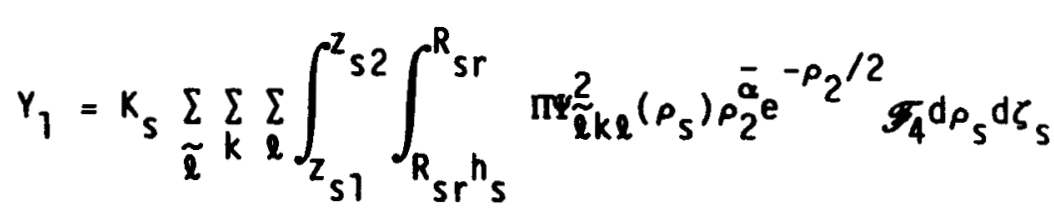

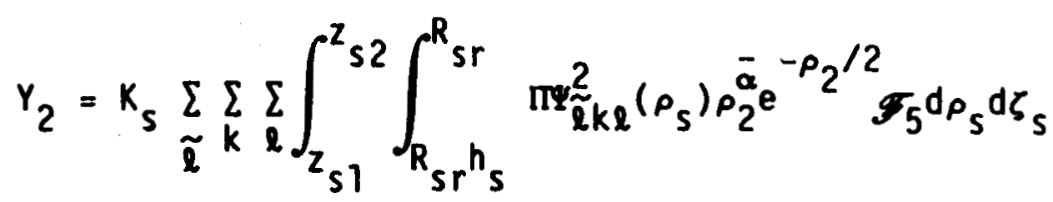

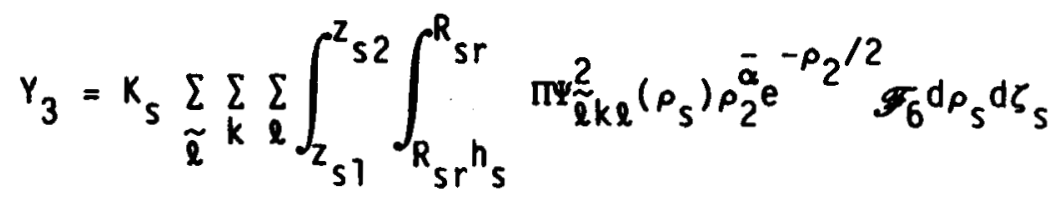

The functions $\mathscr{F}_{1}, \mathscr{F}_{2}, \mathscr{F}_{3}, \mathscr{F}_{4}, \mathscr{F}_{5}, \mathscr{F}_{6}, K_{\mathrm{r}}, K_{5}$ are defined in the Appendix. Figure $A 1$ shows the angles $\bar{\varphi}_{r}$, $\varphi_{r}$ for the rotor and 1.jkewise $\bar{\varphi}_{s}$, $\varphi_{S}$ on the stator. If $R_{i}\left(r_{1}, \theta_{1}, z_{1}\right)$ and $s_{1}\left(r_{1}, \theta_{1}, z_{1}\right)$ denote the vector positions of a set of points on the rotor and stator, the respective Birnbaum coefficients are determined by satisfying the boundary conditions at these points on both the upper and lower surfaces. If $z^{\prime}=z^{\prime}\left(y^{\prime}\right)$ is the equation of the blade profile in the blade coordinate system, the flow tangency condition is expressed as $\tau=\left(d z^{\prime} / d y^{\prime}\right)=\left(U_{z^{\prime}} / U y^{\prime}\right)$. Denoting by $\mathscr{Z}^{\prime}=0+$ and $\mathscr{Z}^{\prime}=0$-, the upper and lower surface chord lines; the boundary conditions for the rotor and the stator may be written as

$$
\begin{array}{ll}
\left(U_{R Z^{\prime}}-\tau_{U 1} U_{R y^{\prime}}\right)_{Z^{\prime}=0+}=0 & \left(U_{R Z^{\prime}}-\tau_{L I} U_{R y^{\prime}}\right)_{Z^{\prime}=0-}=0 \\
\left(U_{S Z^{\prime}}-\tau_{U 2} U_{S Y^{\prime}}\right)_{Z^{\prime}=0+}=0 & \left(U_{S Z^{\prime}}-\tau_{L 2} U_{S y^{\prime}}\right)_{Z^{\prime}=0-}=0
\end{array}
$$

where $\tau_{U l}, \tau_{L I}$ are the chordwise slopes of the upper and lower surfaces at a point on a rotor blade; $\tau_{U 2}$ and $\tau_{L 2}$ represent the corresponding quantities at points on a stator blade. Substituting from equation (3.1) we can write these equations as 


$$
K_{\mathscr{A}}^{(0)}=\mathscr{C}_{1} \quad \mathrm{LAd}^{(0)}=\mathscr{C}_{2} \quad \mathrm{U}_{\mathscr{B}}{ }^{(0)}=\mathscr{C}_{3} \quad \mathrm{~V}_{\mathscr{B}}{ }^{(0)}=\mathscr{C}_{4}
$$

which can be rewritten as

$$
\begin{array}{lll}
\mathrm{K}_{\mathrm{L}} \mathscr{A}^{(0)}=r_{1} & \mathrm{~K}_{\mathrm{L}}=\left(\begin{array}{l}
\mathrm{K} \\
\mathrm{L}
\end{array}\right) & \mathrm{r}_{1}=\left(\begin{array}{l}
\mathscr{C}_{1} \\
\mathscr{C}_{2}
\end{array}\right) \\
U_{\mathrm{V}} \mathscr{B}^{(0)}=r_{2} & U_{\mathrm{V}}=\left(\begin{array}{l}
U \\
\mathrm{~V}
\end{array}\right) & \mathrm{r}_{2}=\left(\begin{array}{l}
\mathscr{C}_{3} \\
\mathscr{C}_{4}
\end{array}\right)
\end{array}
$$

The matrices $K, L, U, V, r_{1}, \Gamma_{2}$ are defined in the Appendix. From equation (3.5) we obtain the basic Birnbaum coefficients of the isolated rotor and stator as:

$$
\mathscr{A}^{(0)}=K_{L}^{-1} r_{1} \quad \mathscr{B}^{(0)}=U_{V}^{-1} r_{2}
$$

It is possible to calculate the aerodynamic parameters of the rotor and stator flow field using the basic coefficients.

\section{THE RECURSION PROCEDURE}

The Birnbaum coefficients of the isolated rotor and stator were determined in section 3. It is possible to calculate the Birnbaum coefficients of the rotor-stator combination using a recurrence procedure to be described in this section. When the rotor and stator are juxtaposed, the perturbation velocities at the rotor and stator blades may be written 


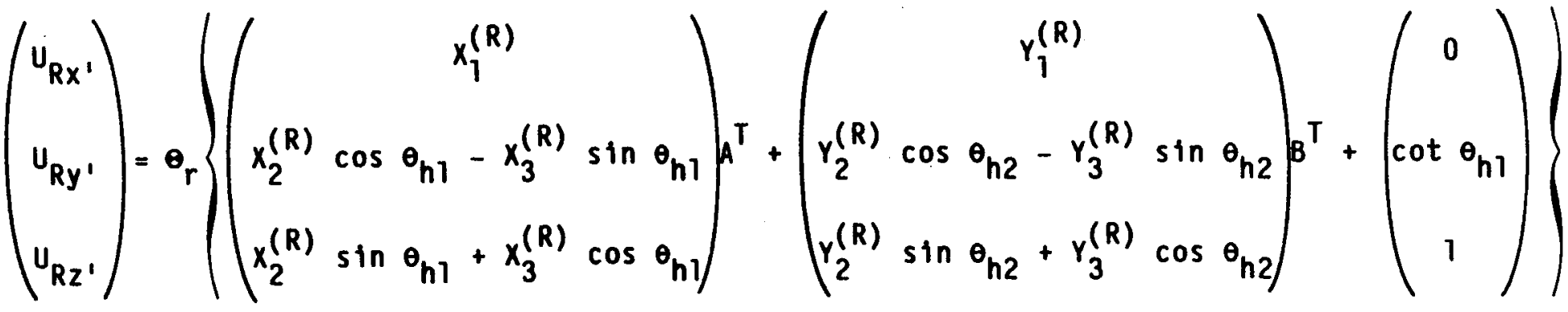

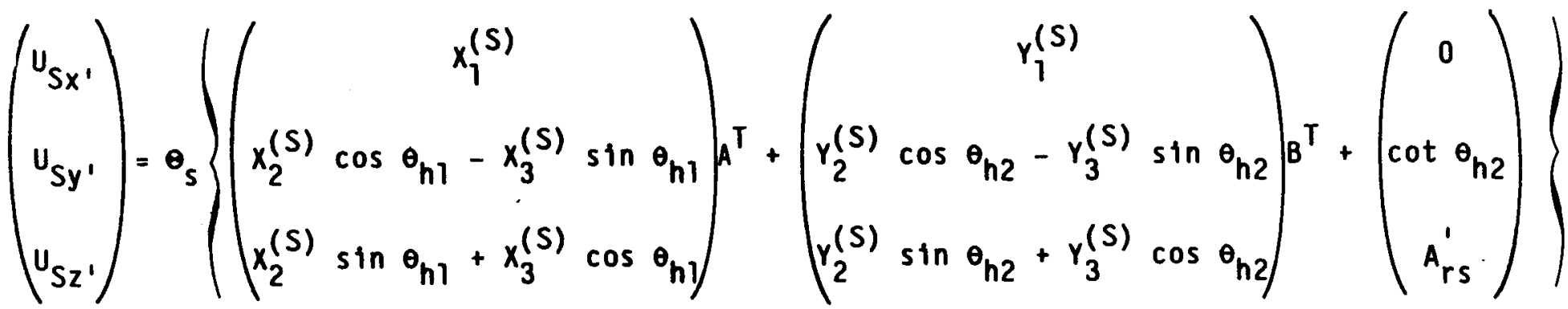

$$
\begin{aligned}
& \theta_{r}=\left(\begin{array}{ccc}
\sin \theta & \cos \theta & 0 \\
\sin \alpha_{r} \cos \theta & -\sin \alpha_{r} \sin \theta & \cos \alpha_{r} \\
-\cos \alpha_{r} \cos \theta & \cos \alpha_{r} \sin \theta & \sin \alpha_{r}
\end{array}\right) \\
& \theta_{s}=\left(\begin{array}{ccc}
\sin \theta & \cos \theta & 0 \\
\sin \alpha_{s} \cos \theta & -\sin \alpha_{s} \sin \theta & \cos \alpha_{s} \\
-\cos \alpha_{s} \cos \theta & \cos \alpha_{s} \sin \theta & \sin \alpha_{s}
\end{array}\right)
\end{aligned}
$$

where $\mathscr{A}$ and $\mathscr{B}$ are the modified Birnbaum coefficients written in series form as

$$
\begin{aligned}
& \mathscr{A}=\mathscr{A}^{(0)}+\mathscr{A}^{(1)}+\mathscr{A}^{(2)}+\ldots \\
& \mathscr{B}=\mathscr{C}^{(0)}+\mathscr{D}^{(1)}+\mathscr{B}^{(2)}+\ldots
\end{aligned}
$$

Using the equations (4.1) and (4.2) in the boundary conditions equation (3.4), we obtain

$$
\begin{aligned}
& \mathrm{K}(\mathscr{A}(0)+\mathscr{A}(1)+\mathscr{A}(2)+\ldots)+\mathrm{s}(\mathscr{B}(0)+\mathscr{B}(1)+\mathscr{B}(2)+\ldots)=\mathscr{C}_{1} \\
& \mathrm{~L}(\mathscr{A}(0)+\mathscr{A}(1)+\mathscr{A}(2)+\ldots)+\mathrm{T}(\mathscr{B}(0)+\mathscr{B}(1)+\mathscr{B}(2)+\ldots)=\mathscr{C}_{2} \\
& \mathrm{M}(\mathscr{A}(0)+\mathscr{A}(1)+\mathscr{A}(2)+\ldots)+\mathrm{U}(\mathscr{B}(0)+\mathscr{B}(1)+\mathscr{B}(2)+\ldots)=\mathscr{C}_{3} \\
& \mathrm{~N}(\mathscr{A}(0)+\mathscr{A}(1)+\mathscr{A}(2)+\ldots)+\mathrm{V}(\mathscr{B}(0)+\mathscr{B}(1)+\mathscr{B}(2)+\ldots)=\mathscr{C}_{4}
\end{aligned}
$$
of equations 


$$
\begin{aligned}
& \left.\left.K_{L} \cos ^{(0)}+\mathscr{A}^{(1)}+\mathscr{A}^{(2)}+\ldots\right)+S_{T}(0)+\mathscr{B}^{(1)}+\mathscr{B}^{(2)}+\ldots\right)=\Gamma_{1}
\end{aligned}
$$

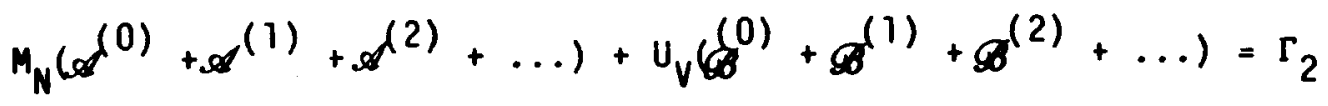

From equation (3.5) we can obtain $\mathscr{A}(0)$ and $\mathscr{G}^{(0)}$ such that equation $(4.4)$ can be reduced to

$$
\begin{aligned}
& \left.K_{L} \mathscr{A}^{(1)}+\mathscr{A}^{(2)}+\mathscr{A}^{(3)}+\ldots\right)+\mathrm{s}_{T}\left(\mathscr{D}^{(1)}+\mathscr{D}^{(2)}+\mathscr{B}^{(3)}+\ldots\right)=-\mathrm{s}_{T} \mathscr{D}^{(0)} \\
& =-\left(S_{T} U_{V}^{-1}\right) r_{2} \\
& \left.M_{N} \mathscr{D}^{(1)}+\mathscr{A}^{(2)}+\mathscr{A}^{(3)}+\ldots\right)+U_{V}\left(\dot{B}^{(1)}+\mathscr{B}^{(2)}+\mathscr{B}^{(3)}+\ldots\right)=-M_{N} \mathscr{D}^{(0)} \\
& =-\left(M_{N} K_{L}^{-1}\right) \Gamma_{1}
\end{aligned}
$$

We set

$$
K_{L} D^{(1)}=-S_{T} \delta^{(0)} \quad U_{V} \delta^{(1)}=-M_{A} d^{(0)}
$$

so that we obtain

$$
\begin{aligned}
& \mathscr{A}^{(1)}=-K_{L}^{-1} S_{T} \mathscr{D}^{(0)}=-K_{L}^{-1} S_{T} U_{V}^{-1} \Gamma_{2} \\
& \mathscr{B}^{(1)}=-U_{V}^{-1} M_{N} \mathbb{P}^{(0)}=-U_{V}^{-1} M_{N} K_{L}^{-1} \Gamma_{1}
\end{aligned}
$$

We substitute these in equation (4.5) and obtain

$$
\begin{aligned}
& \left.\mathrm{K}_{\mathrm{L}} \operatorname{\phi d}^{(2)}+\mathscr{A}^{(3)}+\mathscr{A}^{(4)}+\ldots\right)+\mathrm{S}_{\mathrm{T}}\left(\mathscr{b}^{(2)}+\mathscr{B}^{(3)}+\mathscr{B}^{(4)}+\ldots\right) \\
& =-S_{T} \oint^{(1)}=+S_{T} U_{V}^{-1} M_{N} K_{L}^{-1} r_{1} \\
& M_{N}\left(\mathscr{D}^{(2)}+\mathscr{A}^{(3)}+\mathscr{A}^{(4)}+\ldots\right)+U_{V}\left(\mathscr{D}^{(2)}+\mathscr{6}^{(3)}+\mathscr{6}^{(4)}+\ldots\right) \\
& =-M_{N} d^{(1)}=+M_{N} K_{L}^{-1} S_{T} U_{V}^{-1} \Gamma_{2}
\end{aligned}
$$

Next put

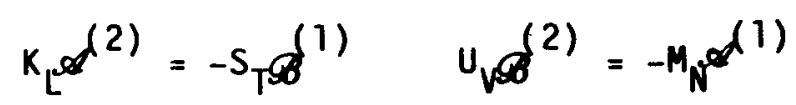


and obtain

$$
\begin{aligned}
& \mathscr{A}^{(2)}=-K_{L}^{-1} S_{T} \mathscr{f}^{(1)}=+K_{L}^{-1} S_{T} U_{V}^{-1} M_{N} K_{L}^{-1} \Gamma_{1} \\
& \mathscr{B}^{(2)}=-U_{V}^{-1} M_{N} \mathscr{d}^{(1)}=+U_{V}^{-1} M_{N} K_{L}^{-1} S_{T} U_{V}^{-1} \Gamma_{2}
\end{aligned}
$$

Substituting into equation (4.8) we get the revised equation

$$
\begin{aligned}
& \left.K_{L} \mathscr{\phi}^{(3)}+\mathscr{d}^{(4)}+\ldots\right)+S_{T}\left(\mathscr{S}^{(3)}+\mathscr{S}^{(4)}+\ldots\right)=-S_{S} \mathscr{S}^{(2)} \\
& M_{N}\left(\mathscr{S}^{(3)}+\mathscr{d}^{(4)}+\ldots\right)+U_{V}\left(\mathscr{S}^{(3)}+\mathscr{S}^{(4)}+\ldots\right)=-M_{N} \mathscr{S}^{(2)}
\end{aligned}
$$

from which using the same procedure as before we can obtain

$$
\begin{aligned}
& \mathscr{A}^{(3)}=-K_{L}^{-1} S_{T} \mathscr{S}^{(2)} \\
& \mathscr{G}^{(3)}=-U_{V}^{-1} M_{N} \mathscr{d}^{(2)}
\end{aligned}
$$

The sequence of Birnbaum coefficients may be written as follows:

$$
\begin{aligned}
& \mathscr{A}^{(0)}=K_{L}^{-1} \Gamma_{1} \quad \mathscr{B}^{(0)}=U_{V}^{-1} \Gamma_{2} \\
& \mathscr{\phi}^{(1)}=-K_{L}^{-1} S \sigma^{(0)} \quad \sigma^{(1)}=-U_{V}^{-1} M_{N} \mathscr{\sigma}^{(0)} \\
& \mathscr{A}^{(2)}=-K_{L}^{-1} S_{T} \mathscr{B}^{(1)} \quad \mathscr{B}^{(2)}=-\mathrm{U}_{V}^{-1} M_{N} \mathscr{A}^{(1)} \\
& \mathscr{A}^{(3)}=-K_{L}^{-1} S_{T} \mathscr{B}^{(2)} \quad \mathscr{G}^{(3)}=-U_{V}^{-1} M_{N} \mathscr{D S}^{(2)} \\
& \text { : } \\
& \mathscr{A}^{(m)}=-K_{L}^{-1} S_{f} \mathscr{S}^{(m-1)} \cdot \mathscr{G}^{(m)}=-U_{V}^{-1} M_{f} \mathscr{f}^{(m-1)}
\end{aligned}
$$

It is observed that the successive terms of the sequence can be generated easily by using the operators $\left(K_{[}^{1} S_{T}\right)$ and $\left(U_{\nabla}^{1} M_{N}\right)$ repeatedly on the result of the previous recursion.

Truncating the Birnbaum serles at $m=M_{*}$, we have $\left(M_{*}+1\right)$ coefficients for each of the rotor and stator. Considering $P_{*}$ points on each side of a blade, we have $2 P_{*}$ equations to determine the $\left(M_{*}+1\right)$ coefficients of the rotor and stator. Therefore, we must have $2 P_{*}=M_{*}+1$. The matrices $K_{L}, M_{N}, S_{T}$, and $U_{V}$ are of order $\left(2 P_{\star} X 2 P_{\star}\right) ;$ The matrices $\Gamma_{1}$ and $\Gamma_{2}$ are of order $\left(2 p_{\star} X 1\right)$. Each of the vectors $\mathscr{A}(0), \mathscr{A}(1), \ldots, \mathscr{B}(0), \mathscr{B}(1), \ldots$ is of order $(2 P * X 1)$. The Birnbaum coefficients may be written as 


$$
\begin{aligned}
& \mathscr{A}=\mathrm{K}_{\mathrm{L}}^{-1}\left(\Gamma_{1}-\mathrm{S}_{\mathrm{P}} \mathscr{\mathscr { B }}=\mathscr{A}^{(0)}-\mathrm{K}_{\mathrm{L}}^{-1} \mathrm{~S}_{\mathrm{T}} \mathscr{B}\right. \\
& \mathscr{B}=\mathrm{U}_{\mathrm{V}}^{-1}\left(\Gamma_{2}-M_{N} \mathscr{O}\right)=\mathscr{B}^{(0)}-U_{\mathrm{V}}^{-1} \mathrm{M}_{\mathrm{N}} \mathscr{A}
\end{aligned}
$$

which clearly shows the coupled nature of the rotor-stator flow and the correction terms to the respective Birnbaum coefficients due to the aerodynamic interference. The structure of the matrices $K, L, M$, and $N$ is given in the Appendix in truncated form along with that of $\mathscr{C}_{7}, \mathscr{C}_{2}, \mathscr{C}_{3}$, and $\mathscr{C}_{4}$. Therefore, the matrices $K_{L}, M_{N}, S_{T}$, and $U_{V}$ along with $\Gamma_{1}$ and $\Gamma_{2}$ are given by the partitioned matrices

$$
\begin{aligned}
& K_{L}=\left(\begin{array}{l}
K \\
L
\end{array}\right) \quad M_{N}=\left(\begin{array}{l}
M \\
N
\end{array}\right) \quad r_{1}=\left(\begin{array}{l}
\mathscr{C}_{1} \\
\mathscr{C}_{2}
\end{array}\right) \\
& s_{T}=\left(\begin{array}{l}
s \\
T
\end{array}\right) \quad U_{V}=\left(\begin{array}{l}
u \\
v
\end{array}\right) \quad r_{2}=\left(\begin{array}{l}
\mathscr{C}_{3} \\
\mathscr{C}_{4}
\end{array}\right)
\end{aligned}
$$

In equation (4.16) we have $K_{\alpha \beta}\left(R_{\alpha}\right), L_{\alpha \beta}\left(R_{\alpha}\right), M_{\alpha \beta}\left(R_{\alpha}\right), N_{\alpha \beta}\left(R_{\alpha}\right), S_{\alpha \beta}\left(S_{\alpha}\right)$, $T_{\alpha \beta}\left(S_{\alpha}\right), U_{\alpha \beta}\left(S_{\alpha}\right), V_{\alpha \beta}\left(S_{\alpha}\right), \mathscr{C}_{1 \alpha}\left(R_{\alpha}\right), \mathscr{C}_{2 \alpha}\left(R_{\alpha}\right), \mathscr{C}_{3 \alpha}\left(S_{\alpha}\right)$, and $\mathscr{C}_{4 \alpha}\left(S_{\alpha}\right)$ corresponding to the points $R_{\alpha}$ and $S_{\alpha}$ on the rotor and stator blade respectively with $\alpha=1,2,3, \ldots P_{\star}, \beta=m+1, m=0,1,2,3, \ldots M_{\star}$. The matrices $K_{L}, M_{N}, S_{T}$, and UV are primarily aerodynamic in nature whlle the matrices $\Gamma_{1}$ and $\Gamma_{2}$ are purely geometric representing the blade section and the stage configuration. The individual elements of the aerodynamic matrices $K, L, M, N, S, T, U, V$, and the geometric configuration matrices $\mathscr{C}_{1}, \mathscr{C}_{2}, \mathscr{C}_{3}$ and $\mathscr{C}_{4}$ have been

defined in the Appendix.

From the simple recurrence procedure outlined above it is possible to obtain the Birnbaum coefficients of the rotor-stator combination to any desired approximation. In the next section we shall outline a direct method for calculating the Birnbaum coefficients for the rotor-stator combination directly.

\section{THE DIRECT METHOD}

In this section we shall obtain the Birnbaum coefficients of the rotorstator combination directly by satisfying the boundary conditions on the blade surfaces of the rotor and the stator simultaneously without resorting to recursion. For this we rewrite equation (4.4) as

$$
\begin{aligned}
& \mathrm{K}_{\mathrm{L}} \mathscr{A}+\mathrm{S}_{\mathrm{T}} \mathscr{B}=\mathrm{r}_{1} \\
& M_{N} \mathscr{A}+\mathrm{U}_{\mathrm{V}} \mathscr{B}=\mathrm{r}_{2}
\end{aligned}
$$

which form a pair of nonhomogeneous simultaneous equations for $\mathscr{A}$ and $\mathscr{B}$. Solving the two equations simultaneousiy, $\mathscr{A}$ and $\mathscr{B}$ are given by 


$$
\begin{aligned}
& \mathscr{A}=\left(S_{T}^{-1} K_{L}-U_{V}^{-1} M_{N}\right)^{-1}\left(S_{T}^{-1} \Gamma_{1}-U_{V}^{-1} \Gamma_{2}\right) \\
& \mathscr{O}=\left(K_{L}^{-1} S_{T}-M_{N}^{-1} U_{V}\right)^{-1}\left(K_{L}^{-1} \Gamma_{1}-M_{N}^{-1} \Gamma_{2}\right)
\end{aligned}
$$

The Birnbaum coefficient vectors $\mathscr{A}$ and $\mathscr{B}$ given by equation (5.2) pertain to the rotor-stator combination. Equation (5.2) gives directly an exact form for the Birnbaum coefficients $\mathscr{A}$ and $\mathscr{B}$ given approximately in equation (4.15). We can relate the coefficients obtained by the two methods. For this we rewrite equation (5.2) using equation (3.5) as

$$
\begin{aligned}
& \mathscr{A}=\left(S_{\mathrm{T}}^{-1} K_{\mathrm{L}}-\mathscr{L}_{\star}\right)^{-1}\left(\mathrm{~S}_{\mathrm{T}}^{-1} \Gamma_{1}-\mathscr{B}^{(0)}\right) \\
& \mathscr{\mathscr { C }}=\left(\mathscr{L}-M_{\mathrm{N}}^{-1} U_{\mathrm{V}}\right)^{-1}\left(\mathscr{A}^{(0)}-M_{\mathrm{N}}^{-1} \Gamma_{2}\right)
\end{aligned}
$$

where $\mathscr{L}$ and $\mathscr{L}_{\star}$ are defined by

$$
\mathscr{L}=K_{L}^{-1} S_{T} \quad \mathscr{L}_{*}=U_{V}^{-1} M_{N}
$$

We can write equation (4.15) in a form simflar to equation (5.3) by decoupling the two equations. Thus, replacing $\mathscr{B}$ in equation (4.15a) by the second equation and solving for $\mathscr{A}$ and similarly replacing $\mathscr{A}$ in equation (4.15b) using the first of equation (4.15) and solving for $\mathscr{B}$ we get

$$
\begin{aligned}
& \mathscr{A}=\left(\mathrm{I}-\mathscr{L}_{\mathscr{L}}\right)^{-1}\left(\mathscr{A}^{(0)}-\mathscr{L} \mathscr{B}(0)\right. \\
& \mathscr{B}=\left(\mathrm{I}-\mathscr{L}_{\star} \mathscr{L}\right)^{-1}\left(\mathscr{B}^{(0)}-\mathscr{L}_{\star \star ⿻}{ }^{(0)}\right)
\end{aligned}
$$

Comparing equations (5.3) and (5.5) we have the following relation

$$
\left(\mathrm{I}-\mathscr{L} \mathscr{L}_{\star}\right)^{-1} \mathscr{L}=\left(\mathscr{L}^{-1}-\mathscr{L}_{\star}\right)^{-1}
$$

between the operators of the recursive method and the direct method. Thus, equation (5.5) may be used to obtain the exact Birnbaum coefficients and $\mathscr{B}$ of the rotor-stator combination using the basic coefficients $\mathscr{A}(0)$ and $\mathscr{G}(0)$ of the isolated rotor and stator. Using the results obtained above, it is possible to estimate the mutual aerodynamic interference between the rotor and the stator. This will be described in the next section.

\section{ESTIMATION OF MUTUAL INTERFERENCE}

In the above, we have calculated two sets of Birnbaum coefficients $\mathscr{A}(0)$, $\mathscr{O}(0)$ and $\mathscr{\mathscr { O }} \mathscr{O}$. 'As mentioned earlier, the coefficients $\mathscr{\mathscr { C }}(0), \mathscr{O}(0)$ pertain' to the isolated rotor and stator and have no aerodynamic interference effects. On the other hand, the coefficients $\mathscr{A}, \mathscr{B}$ pertain to the combined rotor-stator 
configuration in which aerodynamic interference effects are present. All the flow field parameters of interest can be calculated using the Birnbaum coefficients appropriate to the configuration considered. We shall define the interference in terms of the axial velocity differences between the two configurations. The perturbation velocity at any point can be written as the sum of the perturbation velocities of the rotor and stator. Assuming for simplicity $\omega_{r}=0$ and using equation (4.1), the perturbation velocity at any point $\left(r_{1}, \theta, 2_{1}\right)$ of the combined flow field can be written

$\mathscr{\nu}\left(r_{1}, \theta, z_{1}, t_{1}\right)=\mathscr{\nu}_{r}+\mathscr{v}_{s}=\left(\begin{array}{c}x_{1} \\ x_{2} \cos \theta_{h_{1}}-x_{3} \sin \theta_{h_{1}} \\ x_{2} \sin \theta_{h_{1}}+x_{3} \cos \theta_{h_{1}}\end{array}\right) e^{i \omega_{s} t}\left(\begin{array}{c}y_{1} \\ y_{2} \cos \theta_{h_{2}}-y_{3} \sin \theta_{h_{2}} \\ y_{2} \sin \theta_{h_{2}}+y_{3} \cos \theta_{h_{2}}\end{array}\right) \boldsymbol{s}$ $=\left(\begin{array}{c}x_{1} \\ x_{2} \cos \theta_{h_{1}}-x_{3} \sin \theta_{h_{1}} \\ x_{2} \sin \theta_{h_{1}}+x_{3} \cos \theta_{h_{1}}\end{array}\right)+\left(\begin{array}{c}y_{1} \\ r_{2} \cos \theta_{h_{2}}-y_{3} \sin \theta_{h_{2}} \\ y_{2} \sin \theta_{h_{2}}+y_{3} \cos \theta_{h_{2}}\end{array}\right) e^{1 \omega_{s} t_{1}}$ $-\left(\begin{array}{c}x_{1} \\ x_{2} \cos \theta_{h_{1}}-x_{3} \cos \theta_{h_{1}} \\ x_{2} \sin \theta_{h_{1}}+x_{3} \sin \theta_{h_{1}}\end{array}\right) \mathscr{L}-e^{i \omega_{s} t_{1}}\left(\begin{array}{c}y_{1} \\ y_{2} \cos \theta_{h_{2}}-y_{3} \sin \theta_{h_{2}} \\ y_{2} \sin \theta_{h_{2}}+y_{3} \cos \theta_{h_{2}}\end{array}\right) \mathscr{L}_{*}$

Denoting the four terms of equation (6.1) by $\mathcal{\nu}_{\text {ro }}, \mathcal{\nu}_{\mathrm{s} 0}, \mathcal{Q}_{i s}, \mathcal{Q}_{\text {ir }}$, the perturbation velocity vector $\mathscr{Q}_{i}$ can be written as

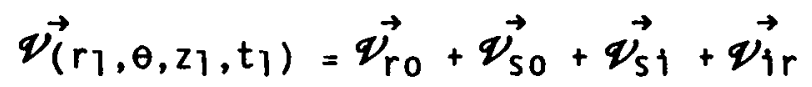

In equation (6.2), the term $\overrightarrow{\mathscr{V}}_{\text {ro }}$ is the perturbation velocity due to the isolated rotor; $\vec{D}_{s}$ is the perturbation velocity due to the isolated stator: $\overrightarrow{\mathcal{Q}_{i s}}$ is the interference velocity produced by the stator on the isolated rotor field; and $\overrightarrow{\mathcal{V}}_{\text {ir }}$ is the interference velocity produced by the rotor on the isolated stator field. The total interference velocity $\mathcal{D}_{i}$ may be written

$$
\vec{q}_{i}=\vec{Q}_{i s}+\vec{q}_{i r}
$$

The interference factor $\varepsilon$ may be defined as the ratio of the axial component of the combined field to the sum of the axial components of the isolated rotor and stator at the same point in the flow. Thus, we write $\varepsilon$ as 


$$
\varepsilon=\frac{\Phi_{1 z}}{\Phi_{\text {roz }}+\Phi_{\text {sOz }}}=\frac{\Phi_{z}}{\Phi_{\text {roz }}+\Phi_{\text {sOz }}}-1
$$

As expected, from this equation we observe that the interference factor $\varepsilon=\left(r_{1}, \theta, z_{1}, t_{1}\right)$ is a point function which is periodic in time.

\section{DISCUSSION}

In the recurrence scheme given above, it is possible to identify the aerodynamic interference effects clearly as opposed to the direct method. Nevertheless, the direct method is also an effictent method for determining the Birnbaum coefficients and thereby the aerodynamic characteristics. Both the methods discussed above enable us to calculate the overall aerodynamic performance of the stage for a given axial and rotational velocity and a stage configuration. The calculation process may be repeated for changes in the velocity and configuration parameters. Thus, it is possible to calculate the perturbation changes for different stator blade angular positions $\varphi_{s}$ for a given rotor blade angle $\varphi_{r}$. Similar calculations for other configuration changes between the rotor and stator may also be made. From a knowledge of the Birnbaum coefficients, it is possible to calculate the distribution of perturbation pressure $\Delta P_{r}$ and $\Delta P_{s}$ on the upper and lower surface of the blades containing the the aerodynamic interference effects

$$
\begin{aligned}
& \Delta p_{r}=-r m^{2}\left(\psi_{z}+\psi_{\theta} \cot \theta_{h_{1}}\right) \\
& \Delta p_{s}=-r m^{2}\left(\psi_{z}+\psi_{\theta} \cot \theta_{h_{1}}\right)
\end{aligned}
$$

We define the dimensionless axial and tangential force coefficients $C_{F Z}$ and $C_{F \theta}$ and the power coefficient $C_{p}$ as

$$
\begin{aligned}
& C_{F Z}=F_{z} /\left(\frac{1}{2} \rho_{\infty} W_{a}^{2} C\right) \\
& C_{F \theta}=F_{\theta} /\left(\frac{1}{2} \rho_{\infty} W_{a}^{2} C\right) \\
& C_{P}=P /\left(\frac{1}{2} \rho_{\infty} W_{a}^{3} R^{2}\right)
\end{aligned}
$$

The axial force coefficients $C_{F R Z}$ and $C_{F S Z}$ per unit blade span per blade of the rotor and stator are given by 


$$
\begin{aligned}
& \frac{d C_{F R Z}}{d r}=-\frac{2}{r} \int_{1}^{2}\left\{\Delta P_{u} \sin \left(\alpha_{r}-\varepsilon_{r u}\right)-\Delta p_{l} \sin \left(\alpha_{r}-\varepsilon_{r l}\right)\right\} \sin \tilde{\omega} d \tilde{\omega} \\
& \frac{d C_{F S Z}}{d r_{1}}=-\frac{2}{r M^{2}} \int_{0}^{\pi}\left\{\Delta P_{u} \sin \left(\alpha_{s}-\varepsilon_{s u}\right)-\Delta p_{l} \sin \left(\alpha_{s}-\varepsilon_{s l}\right)\right\} \sin \tilde{\omega} d \tilde{\omega}
\end{aligned}
$$

while the tangential force coefficients $C_{F R \theta}$ and $C_{F S \theta}$ per unit blade length per blade are given by

$$
\begin{aligned}
& \frac{d C_{F R \theta}}{d r_{1}}=-\frac{2}{r M^{2}} \int_{0}^{\pi}\left\{\Delta P_{u} \cos \left(\alpha_{r}-\varepsilon_{r u}\right)-\Delta P_{\ell} \cos \left(\alpha_{r}-\varepsilon_{r \ell}\right)\right\} \sin \tilde{\omega} d \tilde{\omega} \\
& \frac{d C_{F R \theta}}{d r_{1}}=-\frac{2}{r M^{2}} \int_{0}^{\pi}\left\{\Delta P_{u} \cos \left(\alpha_{s}-\varepsilon_{s u}\right)-\Delta p_{\ell} \cos \left(\alpha_{s}-\varepsilon_{s \ell}\right)\right\} \sin \tilde{\omega} \tilde{d \omega}
\end{aligned}
$$

The power coefficient $C_{p}$ of the rotor is given by

$C_{P}=-\frac{2 C_{R}}{R_{\star} \gamma M^{2}} \int_{h r}^{1} \int_{0}^{\pi}\left\{\Delta P_{u} \cos \left(\alpha_{r}-\varepsilon_{r u}\right)-\Delta p_{\ell} \cos \left(\alpha_{r}-\varepsilon_{r \ell}\right)\right\} \sin \tilde{\omega} d \tilde{\omega} d r_{1}$

$\varepsilon_{r u}=\tan ^{-1} \tau_{U 1} \quad \varepsilon_{r \ell}=\tan ^{-1} \tau_{L 1} \quad \varepsilon_{s u}=\tan ^{-1} \tau_{U 2} \quad \varepsilon_{s l}=\tan ^{-1} \tau_{L 2}$

The upwash velocity of the rotor-stator combination is given by the velocity component $\mathcal{V}_{\tau}$ and may be written as

$$
\mathscr{\nu}_{\tau}=\mathscr{\nu}_{\tau 1}+\mathscr{\nu}_{\tau 2}=\mathrm{X}_{3}+\mathrm{Y}_{3} \mathscr{B}
$$

The circulation distribution over the blade span can be obtained by integrating the pressure normal to the blade chord and is given by

$$
\begin{aligned}
& \Gamma_{R}=-\frac{C_{R}}{r M^{2}} \int_{0}^{\pi}\left(\Delta P_{u}-\Delta P_{\ell}\right)_{R} \sin \tilde{\omega} d \tilde{\omega} \\
& \Gamma_{S}=-\frac{C_{S}}{r M^{2}} \int_{0}^{\pi}\left(\Delta P_{u}-\Delta P_{\ell}\right)_{S} \sin \tilde{\omega} d \tilde{\omega}
\end{aligned}
$$




\section{CONCLUSIONS AND SUMMARY}

Using an acceleration potential method and the lifting surface theory, two methods, a recursive and a direct method, for solving the mutually interacting aerodynamic problem of rotor-stator combination have been outlined for determining the blade loading and other characteristics of an axial compressor stage with the rotor placed ahead of the stator and each with a finite number of blades. Expressions have been given for the resultant pressure field of the rotor and stator separately for an unknown distribution of surface pressure loading on the blades. The blade surface loading has been represented by a modified Birnbaum-Glauert series with unknown coefficients. In the recursive method, a set of basic Birnbaum coefficients is obtained by satisfying the condition of flow tangency on both sides of the rotor and stator blades individually. The successive values of these coefficients are obtained to any desired accuracy by a systematic matrix procedure on the basic set of coefficients to account for the effects of rotor-stator interference. In the direct method, on the other hand, the final values of the Birnbaum coefficients are determined by a single matrix operation satisfying all the surface flow tangency conditions simultaneously. The relation between the two methods has been shown by relating the corresponding matrix operators. The Birnbaum coefficients and $\mathscr{B}$ of the rotor-stator system have been related through the matrix operators to the basic Birnbaum coefficients $\mathscr{S}(0)$ and $\mathscr{B}(0)$ of the isolated rotor and stator. Expressions have been given for calculating the perturbation velocities, the circulation distribution along the blades, the axial and tangential forces on the blades and the power required by the rotor, using these coefficients. 
NOMENCLATURE

$\bar{A}$

$\mathbf{a}_{\boldsymbol{\infty}}$

$\overline{\mathrm{B}}$

C

$\bar{c}$

$\overline{0}$

$h_{r}, h_{s}$

$J_{k}(r)$

$k_{r}, k_{s}$

$L_{a}^{(b)}(r)$

$M$

$\bar{M}$

$\mathrm{M}_{2}$

$M$ *

$P_{r}, P_{s}$

$\overrightarrow{a r}_{r}(r)$ ol $\left.\tau 1\right)$

$\vec{q}_{s}(r 2$, or $r 2)$

$R *=M / M$

$R_{s r}=r_{t s} / r_{t r}$

$\vec{r}(r, \theta, z)$

$r_{1}=r / r_{t r}$

$r_{2}=v \widetilde{\ell} \ell r_{1}$

rhr, rhs

$r_{t r}, r_{t s}$

$t$ arbitrary constant of integration equation (3.1)

free stream speed of sound

arbitrary constant of integration equation (3.1)

blade chord

arbitrary constant of integration equation (3.1)

arbitrary constant of integration equation (3.1)

rotor and stator (hub/tip) radius ratio

Bessel function of the first kind equation (2.3)

rotor and stator circumferential mode numbers

generalized Laguerre function, of degree $a$ and order $b$ equation (2.3)

Mach number of axial flow velocity $=W_{a} / a_{\infty}$

rotor tip Mach number $=\Omega r_{t r} / a_{\infty}$

Mach number of swirl component at rotor exit

number of Birnbaum series coefficients considered

radial pressure functions of rotor and stator

perturbation velocities of the rotor in the helical coordinate system

perturbation velocities of the stator in the helical coordinate system

characteristic radius of rotor

stator tip radius/rotor tip radius

position vector of a point in cylindrical coordinates

dimensionless radial coordinate

radius parameter defined in equation (2.3)

hub radius of rotor and stator

tip radius of rotor and stator

time 


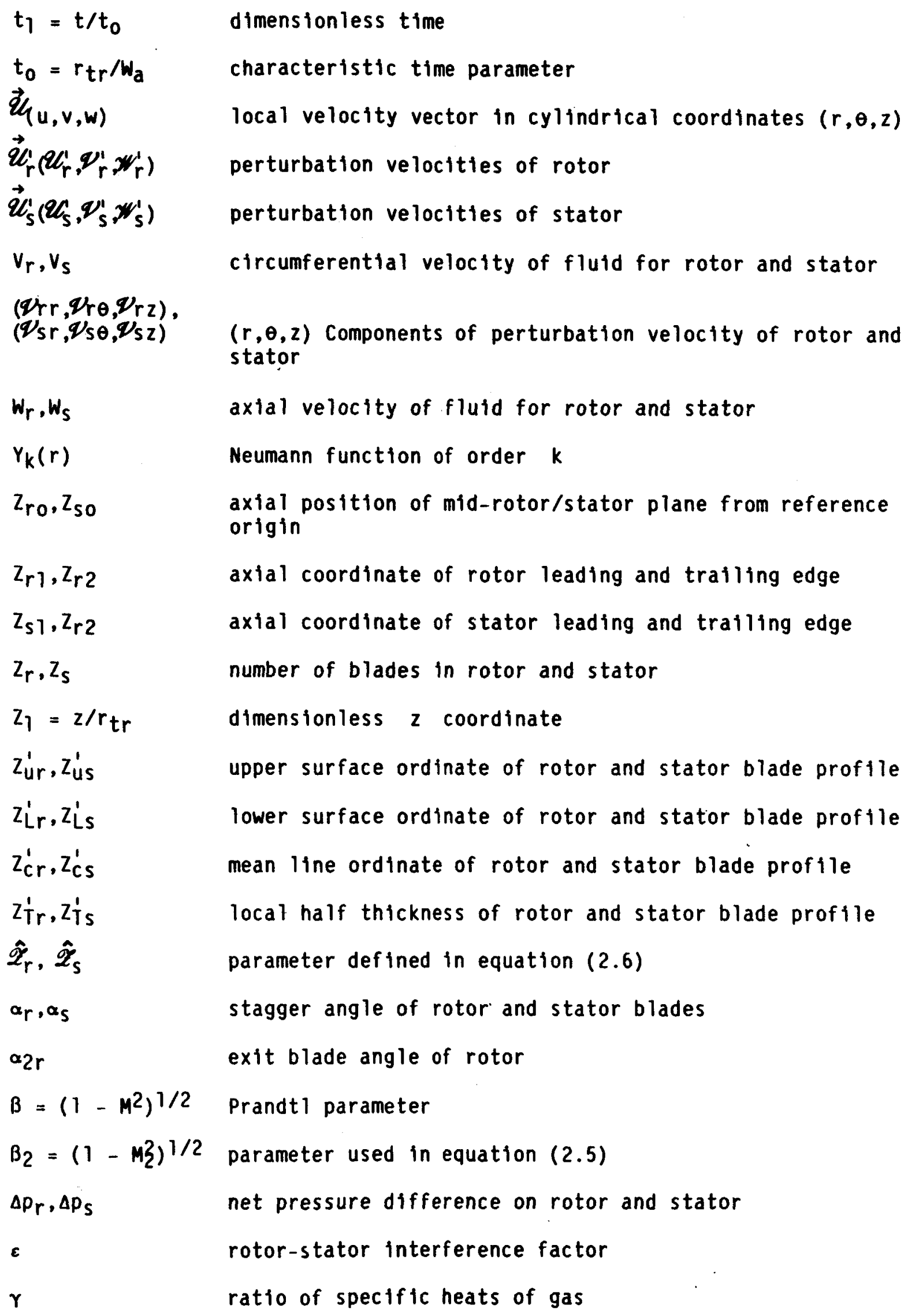


$\Gamma_{r}, \Gamma_{s}$

$\varphi_{r}, \varphi_{s}$

$\varphi_{r}, \varphi_{s}$

$\Phi_{k \ell}(r)$

$\Phi *$

$\Psi_{\ell k \ell}(r)$

$\Psi$ *

Pros

$\omega_{r}, \omega_{s}$

$\lambda_{k l}$

$\Lambda_{k \ell}$

$v \tilde{\ell} k \ell$

$\zeta_{r}, \zeta_{s}$

$\widetilde{\omega}_{r}, \widetilde{\omega}_{s}$

$\Omega$

Subscripts:

$\mathrm{Re} / \mathrm{Im}$

$r, s$

$n, t$

$\mathfrak{i}, j, k$

$r, \theta, z$

$r, \sigma, \tau$
Azimuth angle of field point in cylindrical coordinate system strength of local rotor/stator bound vortex

azimuth angle of pressure pole/dipole on rotor, stator blade mean offset angle of first rotor and stator blade

normalized rotor eigenfunction of mode number $k$ and $P$-th efgenvalue

rotor subsonic pressure function equation (4.10)

normalized stator eigenfunction of order $k$, degree $\tilde{l}$ and $\ell$-th eigenvalue

stator subsonic pressure function

radial position of pressure pole/dipole on rotor, stator blade

Glauert angle of blade defined in equation (2.9)

1-th radial eigenvalue of rotor of circumferential mode number $k$

1-th rotor axial eigenvalue of mode number $k$

stator radial eigenvalue

axial coordinate of rotor/stator pressure pole

angular frequency of oscillating rotor/stator pressure pole ( $\mathrm{rad} / \mathrm{sec})$

angular velocity of rotor

real/imaginary part

rotor, stator

hub, tip

unit vectors along $(x, y, z)$ directions

cylindrical coordinates

local helical coordinates 
The functions $F_{1}, \ldots \ldots \mathscr{F}_{6}$ in equation (3.2) and the coefficients $K_{r}$ and $K_{s}$ contained in it are defined by the following equation (A-1)

$$
\begin{aligned}
& f_{0}=\frac{M \hat{M}}{B^{2}}+1 \frac{A_{K l}}{B} \operatorname{sgn} \hat{Z}_{r} \\
& f_{1}=\int_{+\infty}^{z_{1}} f_{0} f_{2} e^{1 T} 1_{1} \\
& f_{2}=\frac{k_{r}}{\rho_{r}}-f_{0} \tan \alpha_{2 r} \\
& f_{3}=\frac{k_{r}}{R_{\star}} \int_{\infty}^{z_{1}} f_{2} e^{i T_{1}} d z_{1} \\
& \sigma_{1}=z_{1} / \sin \theta_{h 1} \\
& \sigma_{2}=\left(\frac{r_{1}}{R_{2^{*}}} \cos \theta_{h 2}+\sin \theta_{h 2}\right) z_{1} \\
& \hat{z}_{r}=z_{1}-\zeta_{r} \\
& T_{0}=-\frac{\Lambda_{k \ell}}{\beta} \tan \alpha_{r} \exp \left\{\left\{\omega_{r} \zeta_{r}+k_{r}\left(\theta-\bar{\varphi}_{r}-\varphi_{r}-\frac{\dot{Z}_{r}}{R_{\star}}\right\}\right.\right. \\
& T_{1}=z_{1}\left(f_{0}+\omega_{r}+\frac{k_{r}}{R_{\star}}\right)-f_{0} \zeta_{r}+k_{r}\left(\theta_{r o}-\bar{\varphi}_{r}-\varphi_{r}\right) \\
& T_{2}=k_{s}\left(\theta_{s 0}+\frac{Z_{1}}{R_{2 \star}}-\bar{\varphi}_{s}-\varphi_{s}\right)-\omega_{s}\left|\hat{Z}_{s}\right|+\omega_{s} \sigma_{2} \sin \theta_{h 2}
\end{aligned}
$$




$$
\begin{aligned}
& T_{3}=k_{s}\left(\theta-\bar{\varphi}_{s}-\varphi_{s}-\frac{\hat{Z}_{s}}{R_{2^{\star}}}\right)+\omega_{s} \sin ^{2} \theta_{h 2}\left(1+\frac{r_{1}}{R_{2^{\star}}} \cot \theta_{h 2}\right) \zeta_{s} \\
& \tan \theta_{\mathrm{hl}}=\mathrm{M} / \mathrm{Mr}_{1} \\
& \mathscr{F}_{1}=\mathscr{F}_{10} g_{1} \\
& \mathscr{F}_{4}=\mathscr{F}_{40} g_{4} \\
& \mathscr{F}_{10}=\frac{d \mathscr{F}_{20}}{d r_{1}}=\frac{\lambda_{k l}}{2 \Lambda_{k \ell} m_{k l}}\left\{A_{k}\left[J_{k-1}\left(\lambda_{k l} r_{1}\right)-J_{k+1}\left(\lambda_{k l} r_{1}\right)\right]+r_{k-1}\left(\lambda_{k l} r_{1}\right)-r_{k+1}\left(\lambda_{k l} r_{1}\right)\right\} \\
& \mathscr{F}_{20}=\Phi_{k \ell}\left(r_{1}\right) / \Lambda_{k \ell} \\
& \mathscr{F}_{40}=\frac{d \mathscr{F}_{50}}{d r_{1}}=\frac{r_{2}^{\bar{\alpha}} e^{-1 / 2} r_{2}}{n_{\tilde{P} k P}}\left\{c_{1}\left[\left(\frac{\tilde{l}+\bar{\alpha}}{r_{2}}-\frac{1}{2}\right) L_{l}^{(2 \bar{\alpha})}\left(r_{2}\right)-\frac{\tilde{l}+2 \bar{\alpha}}{r_{2}} L_{\ell-1}^{(2 \bar{\alpha})}\left(r_{2}\right)\right]\right. \\
& \left.+r_{2}^{-2 \bar{\alpha}}\left[\left(\frac{\bar{\alpha}}{r_{2}}-\frac{1}{2}\right){ }_{1} F_{1}\left(-\tilde{\ell}-2 \bar{\alpha} ; 1-2 \bar{\alpha} ; r_{2}\right)-\frac{2 \bar{\alpha}}{r_{2}} r_{1}\left(-\tilde{\ell}-2 \bar{\alpha} ;-2 \bar{\alpha} ; r_{2}\right)\right]\right\} \\
& f_{4}=\frac{\omega_{s} \tan \alpha_{s}}{k_{s}} e^{T^{\top} 3} \\
& f_{6}=\frac{i k_{5}}{R_{2^{*}}} \int_{\infty}^{Z_{1}} f_{5} e^{i T_{2}} d z_{1} \\
& g_{1}=\int_{\infty}^{z_{1}} f_{2} e^{i T_{1}} d z_{1} \\
& g_{3}=\frac{i \cos 2 \theta_{h l}}{\cos \theta_{h 1}}\left(T_{0}+f_{1}+f_{3}\right) \\
& g_{5}=\sin \theta_{h 2}\left(f_{5} e^{i T}-i \omega_{s} g_{4}\right)
\end{aligned}
$$

22 


$$
\begin{aligned}
& \theta_{\text {ro }}=\theta-\frac{z_{1}}{R_{\star}} \\
& \hat{z}_{s}=z_{1}-\zeta_{s} \\
& \tan \theta_{h 2}=M /\left(M r_{1}-M_{2}\right)=\left(\cot \theta_{h 1}-\tan \alpha_{2 r}\right)^{-1} \\
& F_{2}=F_{20} g_{2} \\
& \mathscr{F}_{5}=\mathscr{F}_{50} g_{5} \\
& F_{50}=\Psi_{\tilde{\ell}_{k \ell}}\left(r_{1}\right) r_{2}^{\bar{\alpha}} e^{-r_{2} / 2} \\
& f_{5}=\frac{1}{\rho_{s}}+\frac{\omega_{s}}{k_{s}} \tan \alpha_{s} s g n \hat{z}_{s} \\
& f_{7}=1 \omega_{s} \int_{\infty}^{Z_{1}} f_{5} \operatorname{sgn} \hat{Z}_{s} e^{i T_{2}} d z_{1} \\
& g_{2}=\sin \theta_{h 1}\left(f_{2} e^{i T_{1}}-1 \omega_{r} g_{1}\right) \\
& g_{4}=\sin ^{2} \theta_{h 2}\left(1+\frac{r_{1}}{R_{2^{*}}} \cot \theta_{h 2}\right) \int_{\infty}^{z_{1}} f_{5} e^{i T_{2}} d z_{1} \\
& g_{6}=\sin ^{3} \theta_{h 2}\left(\frac{r_{1}}{R_{2^{\star}}} \cot ^{2} \theta_{h 2}-\frac{R_{2^{\star}}}{r_{1}}\right)\left(f_{4}+f_{6}-f_{7}\right) \\
& \theta_{s o}=\theta-\frac{Z_{1}}{R_{2^{\star}}} \quad \Lambda_{k \ell}^{2}=\lambda_{k \ell}^{2}-\frac{\hat{M}^{2}}{B^{2}} \\
& R_{2 \star}=\frac{R_{\star}}{1-R_{\star} \tan \alpha_{2 r}}=\frac{M}{M-M_{2}}
\end{aligned}
$$




$$
\begin{aligned}
& K_{r}=\frac{z_{r} e^{i \omega_{r} t}}{4 \pi \gamma \beta M^{2}} e^{-i \omega_{r} \sigma_{1} \sin \theta_{h 1}} \\
& K_{s}=-\frac{z_{s} e^{i \omega_{s} t}}{8 \pi \gamma M^{4} \tan \alpha_{2 r}} e^{-1 \omega_{s} \sigma_{2} \sin \theta_{h 2}}
\end{aligned}
$$

$$
\begin{array}{ll}
R_{\star}=M / \bar{M} & \hat{M}=M \omega_{r}+\bar{M} k_{r} \\
\mathscr{F}_{3}=M_{20} g_{3} & \\
\mathscr{F}_{6}=\mathscr{F}_{50} g_{6} &
\end{array}
$$


The matrices $K, L, M, N, S, T, U$, and $V$ are expressed by a linear combination of the functions $X_{1}, x_{2}, X_{3}, Y_{1}, Y_{2}$, and $Y_{3}$ using the coefficients $A_{i j}$. The matrices $K, L, M, N, S, T, U, V$, and coeffictents $A_{1 j}$ are given in equations $(A-2)$ and $(A-3)$ as follows:

$$
\begin{array}{ll}
K=f_{1}=\bar{A}_{11} X_{1}+\bar{A}_{12} X_{2}+\bar{A}_{13} X_{3} & S=g_{1}=\bar{A}_{14} Y_{1}+\bar{A}_{15} Y_{2}+\bar{A}_{16} Y_{6} \\
L=f_{2}=A_{21} X_{1}+A_{22} X_{2}+A_{23} X_{3} & T=g_{2}=A_{24} Y_{1}+A_{25} Y_{2}+\bar{A}_{26} Y_{6} \\
M=f_{3}=\bar{A}_{31} X_{1}+\bar{A}_{32} X_{2}+\bar{A}_{33} X_{3} & U=g_{3}=\bar{A}_{34} Y_{1}+\bar{A}_{35} Y_{2}+\bar{A}_{36} Y_{6} \\
N=f_{4}=A_{41} X_{1}+A_{42} X_{2}+\bar{A}_{44} X_{4} & V=g_{4}=A_{44} Y_{1}+\bar{A}_{45} Y_{2}+\bar{A}_{46} Y_{6}
\end{array}
$$

$$
\begin{aligned}
& \bar{A}_{11}=\cos \theta\left(\tau_{U 1} \sin \alpha_{r}+\cos \alpha_{r}\right) \\
& \bar{A}_{12}=\sin \theta_{h 1}\left(\tau_{U 1} \cos \alpha_{r}-\sin \alpha_{r}\right)-\sin \theta \cos \theta_{h 1}\left(\tau_{U 1} \sin \alpha_{r}+\cos \alpha_{r}\right) \\
& \bar{A}_{13}=\cos \theta_{h 1}\left(\tau_{U 1} \cos \alpha_{r}-\sin \alpha_{r}\right)+\sin \theta \sin \theta_{h 1}\left(\tau_{U 1} \sin \alpha_{r}+\cos \alpha_{r}\right) \\
& \bar{A}_{14}=\cos \theta\left(\tau_{U 1} \sin \alpha_{r}+\cos \alpha_{r}\right) \\
& \bar{A}_{15}=\sin \theta_{h 2}\left(\tau_{U 1} \cos \alpha_{r}-\sin \alpha_{r}\right)-\sin \theta \cos \theta_{h 2}\left(\tau_{U 1} \sin \alpha_{r}+\cos \alpha_{r}\right) \\
& \bar{A}_{16}=\cos \theta_{h 2}\left(\tau_{U 1} \cos \alpha_{r}-\sin \alpha_{r}\right)+\sin \theta \sin \theta_{h 2}\left(\tau_{U 1} \sin \alpha_{r}+\cos \alpha_{r}\right) \\
& \mathscr{C}_{1}=\sin \theta \cot \theta_{h 1}\left(\tau_{U 1} \sin \alpha_{r}+\cos \alpha_{r}\right)-\left(\tau_{U 1} \cos \alpha_{r}-\sin \alpha_{r}\right) \\
& \bar{A}_{31}=\cos \theta\left(\tau_{U 2} \sin \alpha_{s}+\cos \alpha_{s}\right) \\
& \bar{A}_{32}=\sin \theta_{h 1}\left(\tau_{U 2} \cos \alpha_{s}-\sin \alpha_{s}\right)-\sin \theta \cos \theta_{h 1}\left(\tau_{U 2} \sin \alpha_{s}+\cos \alpha_{s}\right) \\
& \bar{A}_{33}=\cos \theta_{h 1}\left(\tau_{U 2} \cos \alpha_{s}-\sin \alpha_{s}\right)+\sin \theta \sin \theta_{h 1}\left(\tau_{U 2} \sin \alpha_{s}+\cos \alpha_{s}\right) \\
& \bar{A}_{34}=\cos \theta\left(\tau_{U 2} \sin \alpha_{s}+\cos \alpha_{s}\right) \\
& \bar{A}_{35}=\sin \theta_{h 2}\left(\tau_{U 2} \cos \alpha_{s}-\sin \alpha_{s}\right)-\sin \theta \cos \theta_{h 2}\left(\tau_{U 2} \sin \alpha_{s}+\cos \alpha_{s}\right) \\
& \bar{A}_{36}=\cos \theta_{h 2}\left(\tau_{U 2} \cos \alpha_{s}-\sin \alpha_{s}\right)+\sin \theta \sin \theta_{h 2}\left(\tau_{U 2} \sin \alpha_{s}+\cos \alpha_{s}\right) \\
& \mathscr{C}_{3}=\sin \theta \cot \theta_{h 2}\left(\tau_{U 2} \sin \alpha_{s}+\cos \alpha_{s}\right)-\left(\tau_{U 2} \cos \alpha_{s}-\sin \alpha_{s}\right)
\end{aligned}
$$




$$
\begin{aligned}
& \bar{A}_{21}=\cos \theta\left(\tau_{L 1} \sin \alpha_{r}+\cos \alpha_{r}\right) \\
& \bar{A}_{22}=\sin \theta_{h 1}\left(\tau_{L 1} \cos \alpha_{r}-\sin \alpha_{r}\right)-\sin \theta \cos \theta_{h 1}\left(\tau_{L 1} \sin \alpha_{r}+\cos \alpha_{r}\right) \\
& \bar{A}_{23}=\cos \theta_{h 1}\left(\tau_{L 1} \cos \alpha_{r}-\sin \alpha_{r}\right)+\sin \theta \sin \theta_{h 1}\left(\tau_{L 1} \sin \alpha_{r}+\cos \alpha_{r}\right) \\
& \bar{A}_{24}=\cos \theta\left(\tau_{L 1} \sin \alpha_{r}+\cos \alpha_{r}\right) \\
& \bar{A}_{25}=\sin \theta_{h 2}\left(\tau_{L 1} \cos \alpha_{r}-\sin \alpha_{r}\right)-\sin \theta \cos \theta_{h 2}\left(\tau_{L 1} \sin \alpha_{r}+\cos \alpha_{r}\right) \\
& \bar{A}_{26}=\cos \theta_{h 2}\left(\tau_{L 1} \cos \alpha_{r}-\sin \alpha_{r}\right)+\sin \theta \sin \theta_{h 2}\left(\tau_{L 1} \sin \alpha_{r}+\cos \alpha_{r}\right) \\
& \mathscr{C}_{2}=\sin \theta \cot \theta_{h 1}\left(\tau_{L 1} \sin \alpha_{r}+\cos \alpha_{r}\right)-\left(\tau_{L 1} \cos \alpha_{r}-\sin \alpha_{r}\right) \\
& \bar{A}_{41}=\cos \theta\left(\tau_{L 2} \sin \alpha_{s}+\cos \alpha_{s}\right) \\
& \bar{A}_{42}=\sin \theta_{h 1}\left(\tau_{L 2} \cos \alpha_{s}-\sin \alpha_{s}\right)-\sin \theta \cos \theta_{h 1}\left(\tau_{L 2} \sin \alpha_{s}+\cos \alpha_{s}\right) \\
& \bar{A}_{43}=\cos \theta_{h 1}\left(\tau_{L 2} \cos \alpha_{s}-\sin \alpha_{s}\right)+\sin \theta \sin \theta_{h 1}\left(\tau_{L 2} \sin \alpha_{s}+\cos \alpha_{s}\right) \\
& \left.\bar{A}_{44}=\cos \theta_{(\tau L 2} \sin \alpha_{s}+\cos \alpha_{s}\right) \\
& \bar{A}_{45}=\sin \theta_{h 2}\left(\tau_{L 2} \cos \alpha_{s}-\sin \alpha_{s}\right)-\sin \theta \cos \theta_{h 2}\left(\tau_{L 2} \sin \alpha_{s}+\cos \alpha_{s}\right) \\
& \bar{A}_{46}=\cos \theta_{h 2}\left(\tau_{L 2} \cos \alpha_{s}-\sin \alpha_{s}\right)+\sin \theta \sin \theta_{h 2}\left(\tau_{L 2} \sin \alpha_{s}+\cos \alpha_{s}\right) \\
& \mathscr{C}_{4}=\sin \theta \cot \theta_{h 2}\left(\tau_{L 2} \sin \alpha_{s}+\cos \alpha_{s}\right)-\left(\tau_{L 2} \cos \alpha_{s}-\sin \alpha_{s}\right)
\end{aligned}
$$


The schematic structure of the matrices $K, L, \ldots U$, and $v$ for determining the $\left(M_{*}+1\right)$ Birnbaum coefficients of the rotor and stator is shown in equation (A-4) below:

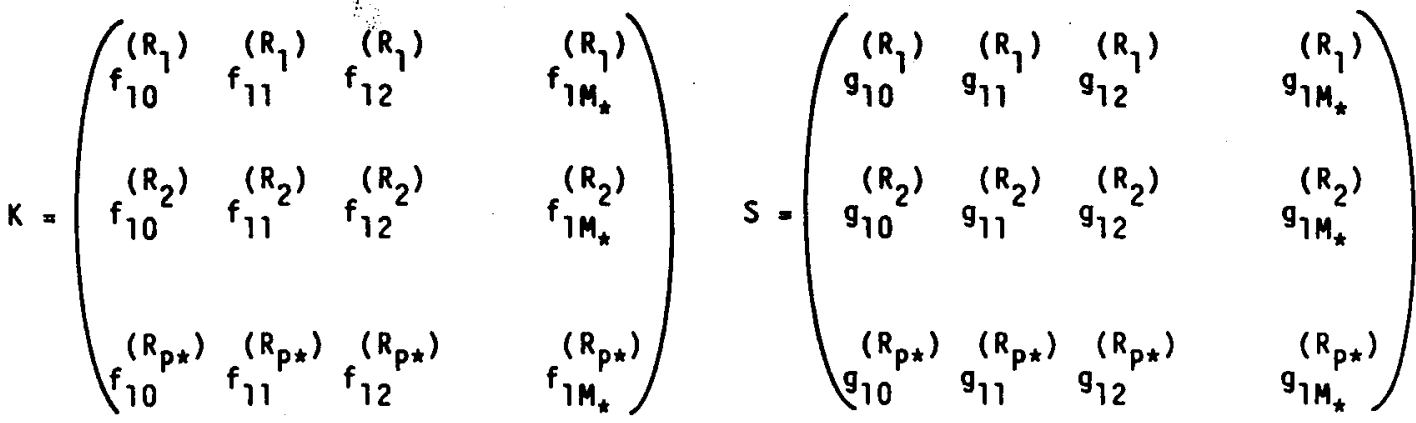

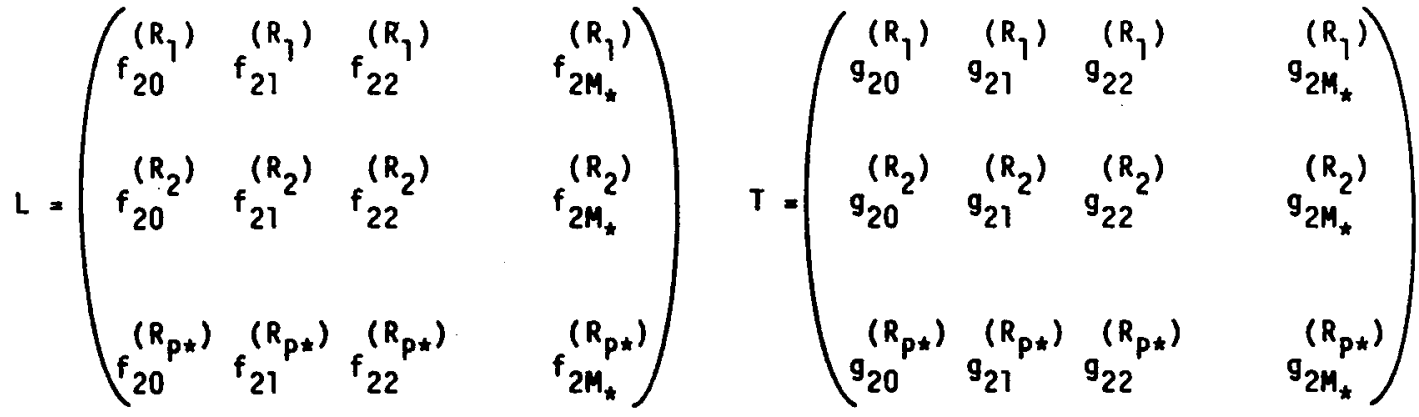

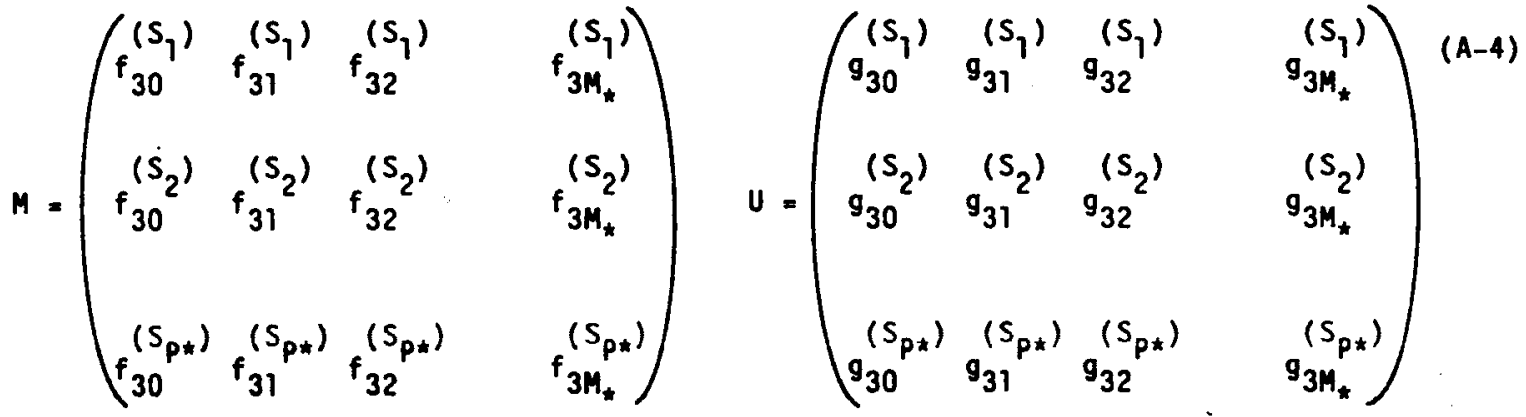

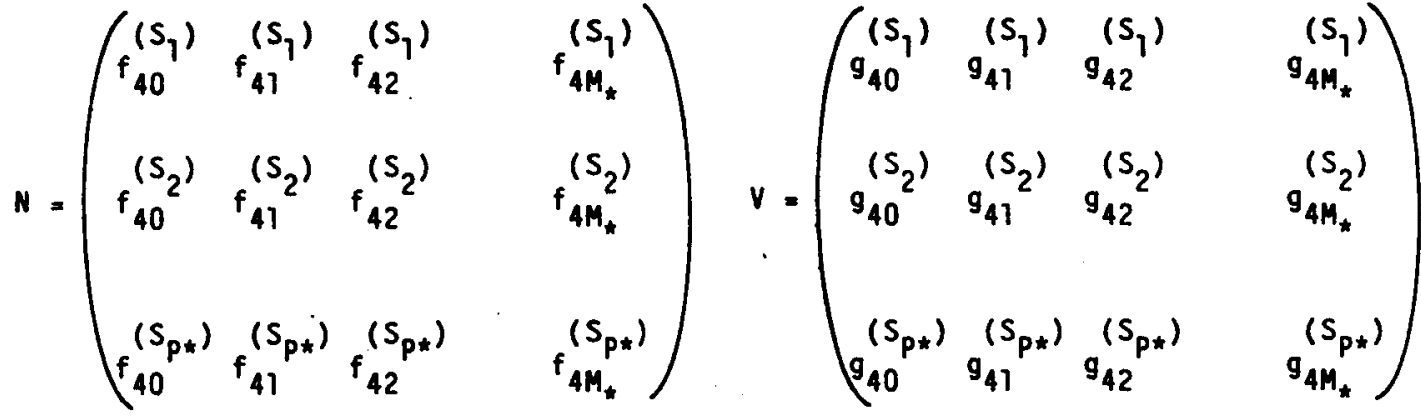

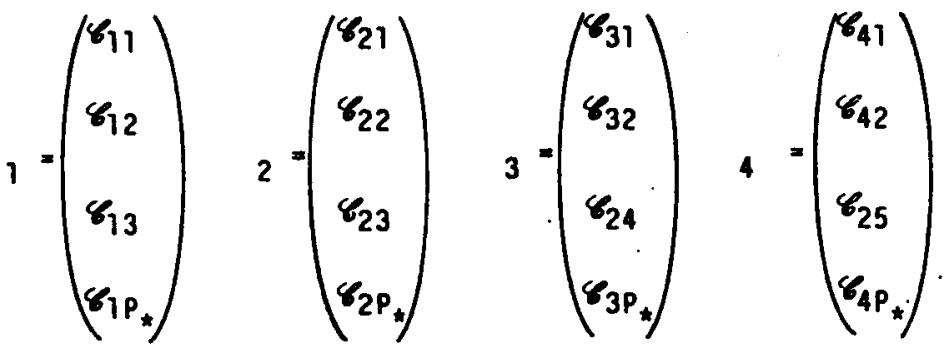




\section{REFERENCES}

1. McCune, J.E., "The Three Dimensional Flow Field of an Axial Compressor Blade Row-Subsonic, Transonic and Supersonic," Ph.D. Thesis, Cornell University, 1958.

2. Namba, M., "Lifting Surface Theory for a Rotating Subsonic or Transonic Blade Row," ARC-R/M-3740, 1974.

3. Schulten, J.B.M.H., "Sound Generated by Rotor Wakes Interacting with a Leaned Vane Stator," AIAA Journal, Vo1. 20, No. 10, Oct. 1982, pp. 1352-1358.

4. Kaji, S. and Okaszaki, T., "Generation of Sound by Rotor-Stator Interaction," Journal of Sound and Vibration, Vol. 13, No. 3, Nov. 1970, pp. 281-307.

5. Ramachandra, S.M., "Acoustic Pressures Emanating From a Turbomachine Stage," AIAA Paper 84-2325, Oct. 1984. (NASA TM-83734). 


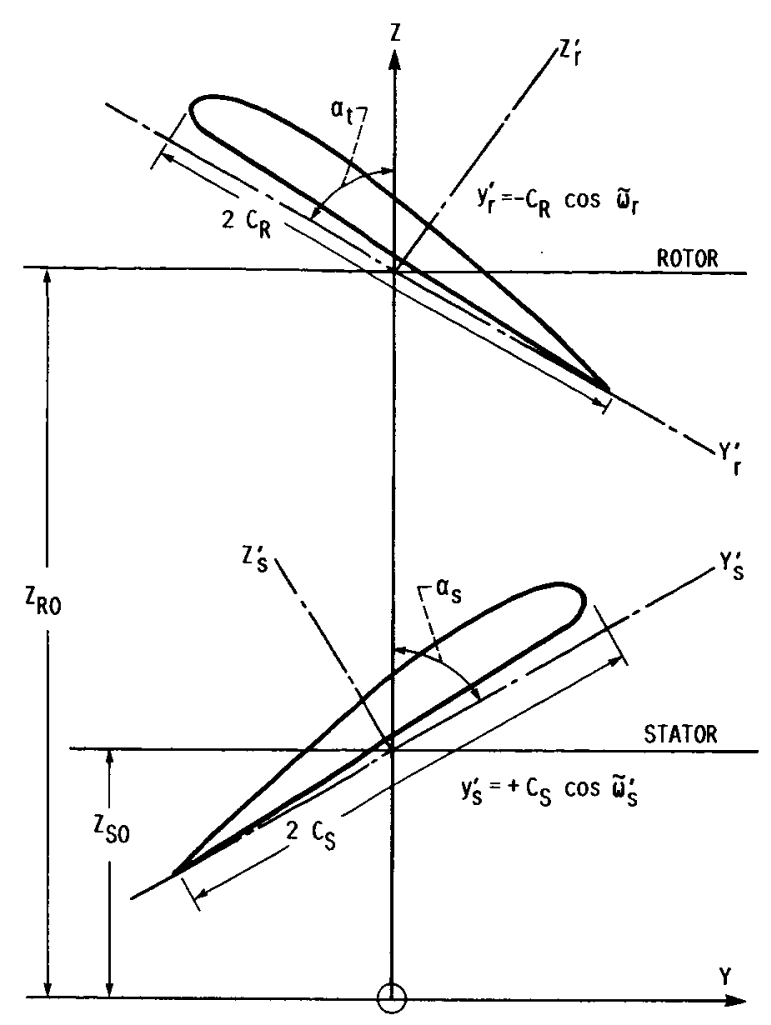

FIGURE 1. - LOCAL COORDINATE SYSTEM FOR BLADES OF STAGE.
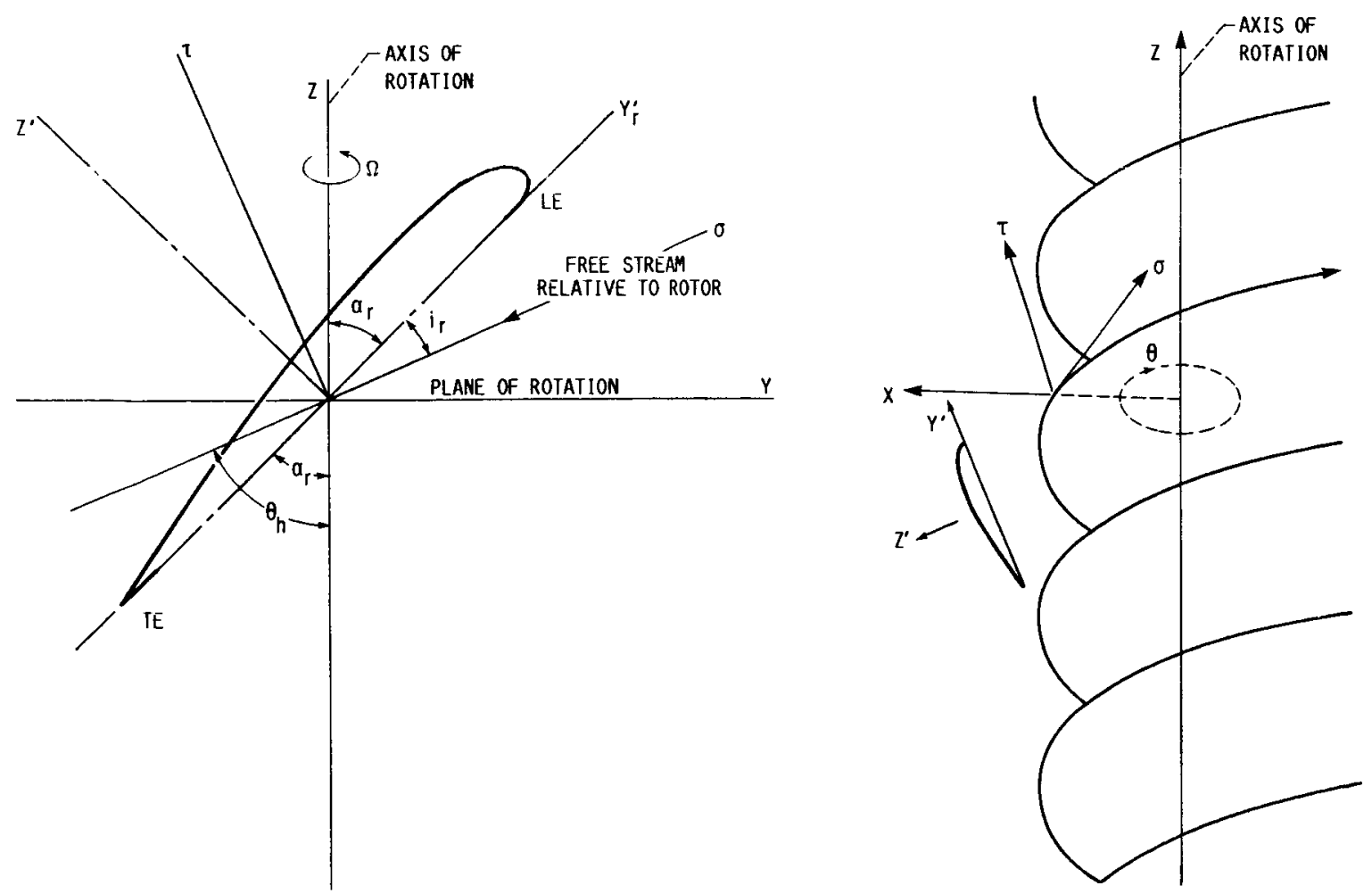

FIGURE 2. - THE HELICAL COORDINATE SYSTEM FOR BLADE. 


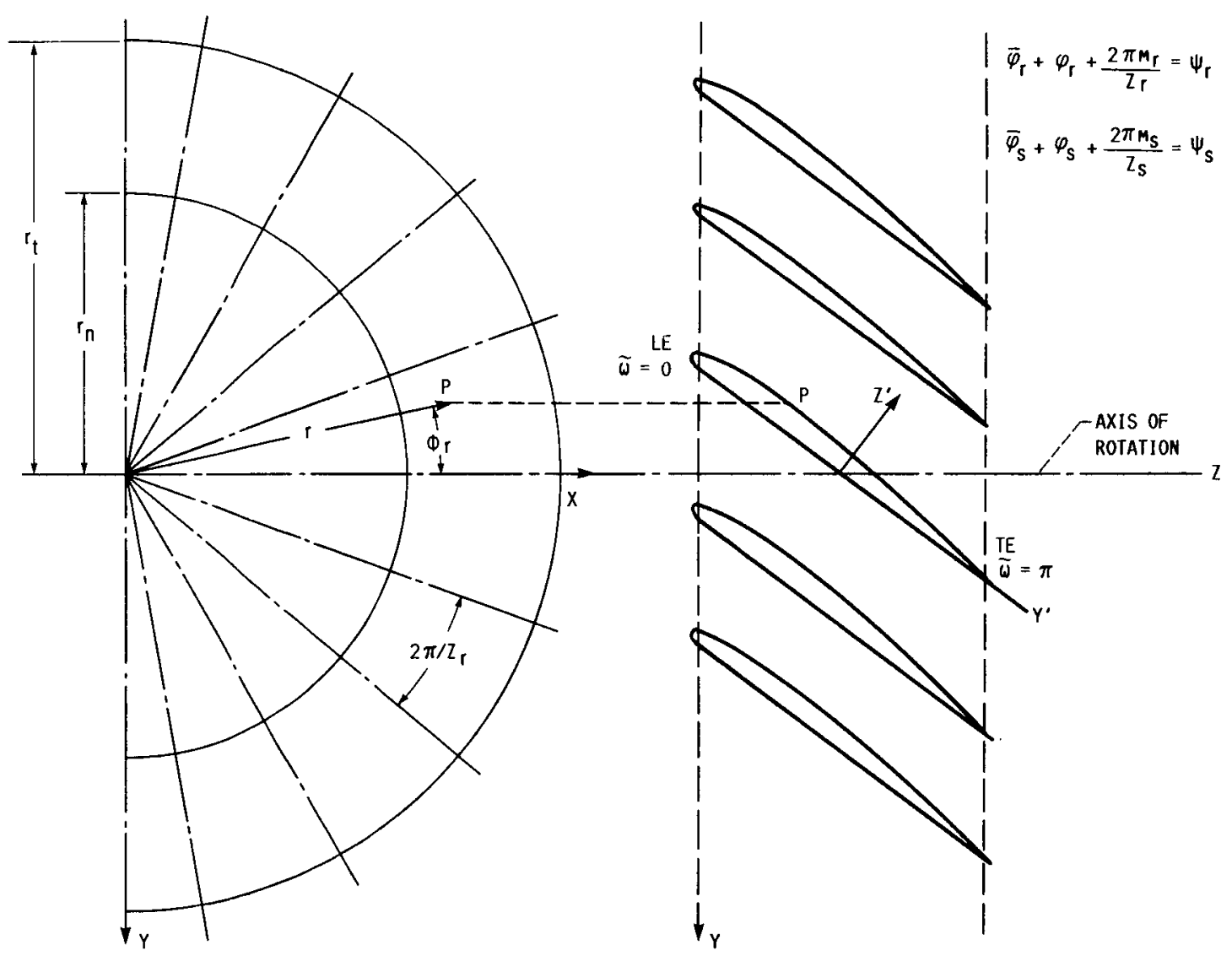

FiguRE 3. - SCHEMAIIC DIAGRAM OF CASCADE. 


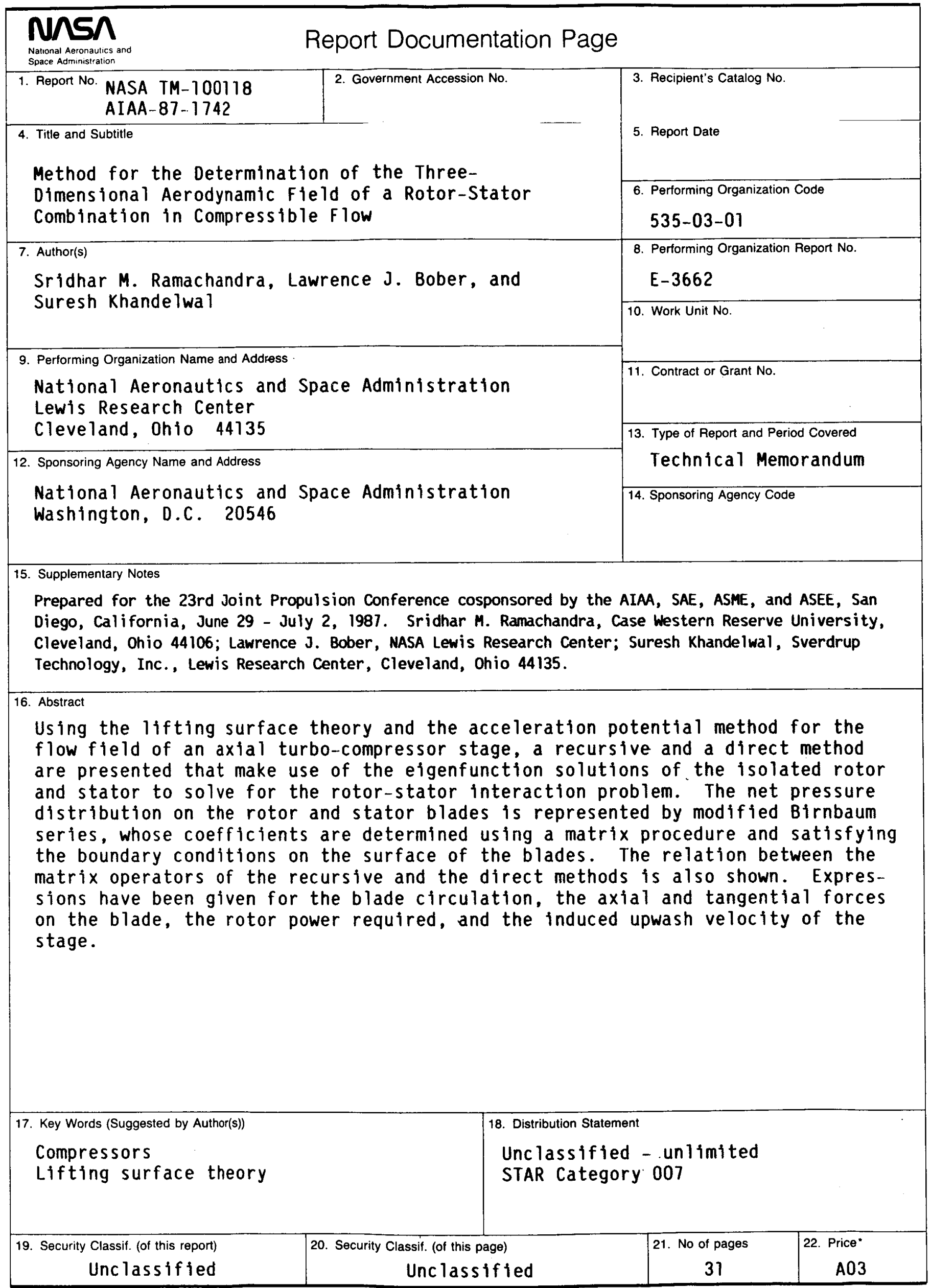

NASA FORM 1626 OCT $86 \quad$ *For sale by the National Technical Information Service, Springfield, Virginia 22161 